\title{
Hunting for ALPs with lepton flavor violation
}

\author{
Claudia Cornella, ${ }^{a, b}$ Paride Paradisi ${ }^{b}$ and Olcyr Sumensari ${ }^{b}$ \\ ${ }^{a}$ Physik-Institut, Universität Zürich, \\ Building 16, Winterthurerstrasse 190, Zürich CH-8057, Switzerland \\ ${ }^{b}$ Istituto Nazionale Fisica Nucleare, Sezione di Padova and \\ Dipartamento di Fisica e Astronomia "G. Galilei", Università degli Studi di Padova, \\ Via Francesco Marzolo, 8, Padova I-35131, Italy \\ E-mail: claudia.cornella@physik.uzh.ch, paride.paradisi@pd.infn.it, \\ olcyr.sumensari@pd.infn.it
}

ABSTRACT: We examine the low-energy signatures of axion-like particles (ALPs) in lepton flavor violating (LFV) processes. By using a dimension-5 effective Lagrangian, we compute the most general ALP contributions to LFV decays of leptons and mesons. The provided expressions are valid for any choice of ALP mass and couplings. We explore the complementarity of different processes, identifying specific patterns to be experimentally tested. Constraints on LFV couplings are derived from existing data and prospects for forthcoming experiments are also discussed. As a by-product, we revisit the possibility of a simultaneous explanation of the observed discrepancies in the muon and electron $g-2$ through ALP interactions.

Keywords: Beyond Standard Model, Effective Field Theories, Heavy Quark Physics, Kaon Physics

ArXiv EPrint: 1911.06279 


\section{Contents}

1 Introduction 1

2 Effective field theory description 3

3 Purely leptonic processes 5

$3.1 \quad \ell_{j} \rightarrow \ell_{i} \gamma$

$3.2 \ell_{j} \rightarrow \ell_{i} \ell_{k} \ell_{k}$

$3.3 \ell_{j} \rightarrow \ell_{i} \gamma \gamma$

$3.4 \mu^{-} \rightarrow e^{-}$conversion in nuclei 10

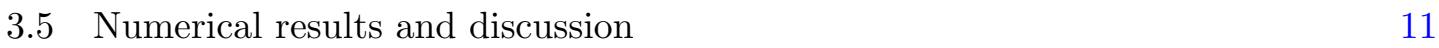

$\begin{array}{ll}3.6 & \text { On the }(g-2)_{e} \text { and }(g-2)_{\mu} \text { anomalies } \\ \end{array}$

4 Hadronic processes 19

$4.1 P \rightarrow \ell_{i} \ell_{j}$ and $\ell_{j} \rightarrow \ell_{i} P \quad 19$

$\begin{array}{lll}\text { 4.1.1 General expressions } & 19\end{array}$

4.1.2 Hadronic inputs: $\mathcal{N}_{P} \quad 20$

$4.2 P \rightarrow P^{\prime} \ell_{i} \ell_{j}$

4.2.1 General expressions $\quad 22$

4.2.2 Hadronic inputs 23

$4.3 \quad P \rightarrow V \ell_{i} \ell_{j}$

4.3.1 General expressions $\quad 23$

4.3.2 Hadronic inputs 24

4.4 Numerical results and discussion 24

5 Conclusions 28

A Useful formulas $\quad \mathbf{3 0}$

A.1 Loop functions 30

A.2 $\ell_{j} \rightarrow \ell_{i} \ell_{k} \ell_{k}$ kinematic functions $\quad 30$

A.3 Form factors for $\ell_{j} \rightarrow \ell_{i} \gamma^{*}$

A.3.1 Linear contributions 31

$\begin{array}{ll}\text { A.3.2 Quadratic contributions } & 32\end{array}$

A.4 Taylor-expanded anapole form factors 32

A.4.1 Linear contributions 33

A.4.2 Quadratic contributions 33

\section{Introduction}

After the long-awaited discovery of the Higgs boson at the Large Hadron Collider (LHC), no convincing evidence of heavy new physics (NP) has emerged yet. The motivation for $\mathrm{NP}$ at the $\mathrm{TeV}$ scale has thus being questioned, and alternative scenarios, with new light mediators, have received increasing attention from both experimental and theoretical com- 
munities over the past several years. A prominent example are Standard Model (SM) extensions with light pseudoscalar bosons, generically referred to as axion-like-particles (ALPs) [1-3]. ALPs might be naturally light, in comparison to the NP scale they originate from, if they are pseudo-Nambu-Goldstone-Bosons (pNGBs) of an underlying broken symmetry. Such particles can be motivated by a number of fundamental open questions in particle physics such as the strong CP problem [4-7], the origin of dark matter [8-11], as well the hierarchy $[12,13]$ and flavor problems [14-17].

Within specific ultraviolet scenarios, the ALP mass and couplings are typically related. Alternatively, a more model independent approach is to consider ALPs as a generalization of the QCD axion, with mass and couplings being free parameters to be probed experimentally. In this context, ALP interactions with SM fermions and gauge bosons are described via an effective Lagrangian built with operators up to dimension-5 [18], and they can be probed in a wide range of experimental facilities. Although less predictive, this approach has the advantage of capturing general features of a broad class of models.

The most stringent limits on ALP couplings arise from cosmological and astrophysical bounds, which are valid for ALP masses below the MeV scale [1-3, 19]. Less severe but still significant bounds are set by LEP for masses ranging from the $\mathrm{MeV}$ scale up to $90 \mathrm{GeV}$ [20]. The possibility of probing ALPs at the LHC, as well as in future colliders and fixedtarget facilities, has also been extensively explored [21-30]. Furthermore, the rich research program at flavor factories offers several opportunities to probe ALP parameters in meson decays [31-33]. In particular, since there is no fundamental reason for the ALP interactions to respect the SM flavor group, ALPs can induce flavor-changing neutral-currents (FCNC) already at tree-level [34-40].

The main goal of this paper is to explore the discovery potential of ALPs through lepton flavor violating (LFV) processes. Since lepton flavor is an accidental symmetry of the SM, broken by the tiny neutrino masses, its observation would correspond to an unambiguous manifestation of new physics. This is an extremely promising and timely subject thanks to the ongoing experimental program at present NA62 [41], LHCb [42] and Belle-II [43], as well as at the future Mu2E [44], Mu3E [45] and COMET [46] experiments, which will improve the current sensitivity by orders of magnitude, see tables 1 and 4 . The main focus in the literature so far has been the on-shell ALP contribution to processes such as $\mu \rightarrow e a \rightarrow e e e$ and $\mu \rightarrow e a \rightarrow e \gamma \gamma$, due to the resonantly enhanced decay rates [47, 48]. For ALP masses above the kinematical threshold of on-shell production, i.e. $m_{a}>m_{\mu}-m_{e}$, there is a nontrivial interplay with loop-induced processes such as $\mu \rightarrow e \gamma$ which has not been fully explored yet. Furthermore, LFV meson decays can provide complementary limits to the ones derived from purely leptonic processes, as they are sensitive to different combinations of ALP couplings.

In this paper, we provide a comprehensive study of ALP-induced LFV decays, generalizing and complementing previous analyses [47-49]. We consider two distinct classes of processes: (i) purely leptonic and (ii) hadronic decays. The first class comprises radiative decays $\ell_{j} \rightarrow \ell_{i} \gamma$, three-body decays $\ell_{j} \rightarrow \ell_{i} \ell_{k} \ell_{k}$ and $\ell_{j} \rightarrow \ell_{i} \gamma \gamma\left(\right.$ with $\left.\ell_{j}=\mu, \tau\right)$, as well as $\mu \rightarrow e$ conversion in nuclei, which we consider to be a leptonic process since the only coherently-enhanced contributions are the ones coming from photon penguins. Hadronic 
decays include leptonic meson decays $P \rightarrow \ell_{i} \ell_{j}$, their inverse processes $\tau \rightarrow P \ell_{j}$, as well as semileptonic decays $P \rightarrow P^{\prime}(V) \ell_{i} \ell_{j}$, where $P^{(\prime)}$ and $V$ stand for pseudoscalar and vector mesons, respectively. We derive general formulae for these processes that can be applied for any choice of the ALP mass and couplings. We show in detail that the relative importance of the various processes depends not only on the relative strength of ALP couplings to photon/gluons and fermions but also on the ALP mass. In particular, the correlations among observables can change drastically depending on whether ALPs are produced on-shell or off-shell. This makes it possible to infer the ALP mass indirectly, through their virtual effects to LFV processes.

Another observable which is highly sensitive to ALP couplings is the anomalous magnetic moment of leptons $a_{\ell}=(g-2)_{\ell} / 2[30,50]$. This quantity received a lot of attention due to the longstanding $\approx 3.6 \sigma$ discrepancy between the experimental measurement and the SM prediction, $\Delta a_{\mu}=a_{\mu}^{\exp }-a_{\mu}^{\mathrm{SM}}=(27.1 \pm 7.3) \times 10^{-10}$ [51-53], which might be soon clarified by the ongoing analysis at the Muon $g-2$ experiment (Fermilab) [54]. In a large class of NP scenarios, contributions to magnetic moment scale with the square of lepton masses (the so-called "naive scaling"), in such a way that the current discrepancy $\Delta a_{\mu}$ suggests a NP effect in $a_{e}$ at the level of $(7 \pm 2) \times 10^{-14}[55] .^{1}$ Testing NP with $(g-2)_{e}$ became possible only very recently thanks to the improved measurement of the fine-structure constant $\alpha_{\mathrm{em}}$ from atomic physics experiments [63]. Remarkably, the reevaluation of $\Delta a_{e}$ employing the latest $\alpha_{\mathrm{em}}$ value has shown a $2.4 \sigma$ deviation from the SM prediction, namely $\Delta a_{e}=(-87 \pm 36) \times 10^{-14}[64,65]$, which departs considerably from the "naive scaling" expectation and has the opposite sign compared to $\Delta a_{\mu}$. Another goal of this paper is to assess to which extent ALPs can explain simultaneously both $g-2$ deviations, exploring the potential interplay with LFV observables (see also ref. [49] for a recent discussion).

The paper is organized as follows. In section 2, we recall the effective description of ALP interactions in terms of dimension-five operators. In section 3, we discuss the purely leptonic probes of LFV ALP couplings, deriving general expressions for the relevant observables and extracting constraints from available experimental results. In section 4, we extend our discussion to LFV processes involving hadrons. Our findings are then summarized in section 5 .

\section{Effective field theory description}

At low energies, the most general dimension-5 effective Lagrangian describing ALP interactions with fermions and photons/gluons reads [18]

$$
\begin{aligned}
\mathcal{L}_{\mathrm{eff}}^{\mathrm{d} \leq 5}= & \frac{1}{2}\left(\partial_{\mu} a\right)\left(\partial^{\mu} a\right)-\frac{m_{a}^{2} a^{2}}{2} \\
& +e^{2} c_{\gamma \gamma} \frac{a}{\Lambda} F_{\mu \nu} \widetilde{F}^{\mu \nu}+g_{s}^{2} c_{g g} \frac{a}{\Lambda} G_{\mu \nu} \widetilde{G}^{\mu \nu}-\frac{\partial_{\mu} a}{\Lambda} \sum_{f, i, j} \bar{f}_{i} \gamma^{\mu}\left(v_{i j}^{f}-a_{i j}^{f} \gamma_{5}\right) f_{j}
\end{aligned}
$$

\footnotetext{
${ }^{1}$ See refs. [55-62] for examples of new physics scenarios that violate the "naive scaling" rule, thus allowing for larger effects in $\Delta a_{e}$ than in $\Delta a_{\mu}$.
} 
where the invariance under the gauge symmetry $\mathrm{SU}(3)_{c} \times \mathrm{U}(1)_{\mathrm{em}}$ has been imposed. In this equation, $f \in\{u, d, \ell\}$ denotes SM fermions in the mass basis, $i, j$ stand for flavor indices and the dual field strengths are defined as $\widetilde{X}^{\mu \nu}=\frac{1}{2} \varepsilon^{\mu \nu \alpha \beta} X_{\alpha \beta}$, with $\varepsilon^{0123}=+1$. The effective couplings to photons and gluons are denoted by $c_{\gamma \gamma}$ and $c_{g g}$, while $v_{i j}^{f}$ and $a_{i j}^{f}$ stand for the vector and axial-vector ALP couplings to SM fermions, and $\Lambda$ for the EFT cutoff. $^{2}$ The matrices $v^{f}$ and $a^{f}$ are taken to be hermitian and real to avoid CP violating effects. By using the equations of motion, the last term in eq. (2.1) can be recast as

$$
\mathcal{L}_{\mathrm{eff}}^{\mathrm{d} \leq 5} \supset-i \frac{a}{\Lambda} \sum_{f, i, j} \bar{f}_{i}\left[\left(m_{f_{j}}-m_{f_{i}}\right) v_{i j}^{f}+\left(m_{f_{j}}+m_{f_{i}}\right) a_{i j}^{f} \gamma_{5}\right] f_{j}
$$

The anomaly equation for the axial-vector current divergence also entails a modification of $c_{\gamma \gamma}$ and $c_{g g}$ couplings, see e.g. discussion in ref. [30]. In the following, we will denote the effective couplings accounting for the full one-loop contributions as $c_{\gamma \gamma} \equiv c_{\gamma \gamma}^{\text {eff }}$ and $c_{g g} \equiv c_{g g}^{\text {eff }}$. From eq. (2.2), we see that $v_{i j}^{f}$ contributes to flavor-violating observables only, as expected from the vector-current conservation, while $a_{i j}^{f}$ enters both flavor-conserving and violating observables. For future convenience, we define the effective couplings $s_{i j}^{\ell}$

$$
s_{i j}^{\ell} \equiv \sqrt{\left|a_{i j}^{\ell}\right|^{2}+\left|v_{i j}^{\ell}\right|^{2}}
$$

which appears in the expressions of most LFV processes, as shown below.

Before studying the phenomenological implications of the effective Lagrangian defined above, we discuss the theoretical bounds on the ALP couplings arising from perturbative unitarity $[66,67]$. Indeed, for sufficiently large values of the energy $\sqrt{s}$, the EFT description is expected to break down. We estimate these constraints by computing the partial wave unitarity bounds on $\gamma \gamma \rightarrow \gamma \gamma, g g \rightarrow g g$ and $\bar{f} f \rightarrow \bar{f} f$ amplitudes mediated by a pseudoscalar boson in the limit of high-energies $\left(\sqrt{s} \gg m_{a}\right)$. By requiring partial waves of total angular momentum $J=0$ to satisfy $\left|\operatorname{Re} a_{0}\right|<1 / 2$, we obtain the following conditions

$$
\begin{aligned}
\sqrt{s} & <\frac{\Lambda}{\sqrt{4 \pi} \alpha_{\mathrm{em}}\left|c_{\gamma \gamma}\right|}, & \sqrt{s} & <\frac{\Lambda}{\sqrt{32 \pi} \alpha_{s}\left|c_{g g}\right|}, \\
\left|v_{i j}^{f}\right| & <\sqrt{\frac{8 \pi}{3}} \frac{\Lambda}{\left(m_{f_{j}}-m_{f_{i}}\right)}, & \left|a_{i j}^{f}\right| & <\sqrt{\frac{8 \pi}{3}} \frac{\Lambda}{\left(m_{f_{j}}+m_{f_{i}}\right)} .
\end{aligned}
$$

As an example, if $\Lambda=1 \mathrm{TeV}$ and $c_{g g}\left(c_{\gamma \gamma}\right)=1$, from eq. (2.4) we learn that our EFT remains unitary up to energies $\sqrt{s} \lesssim 10(40) \mathrm{TeV}$. On the other hand, bounds on the Yukawa couplings $v_{i j}^{f}$, and $a_{i j}^{f}$ do not depend on $\sqrt{s}$. For instance, for $\Lambda=1 \mathrm{TeV}$, eq. (2.5) yields $\left|v_{3 i}^{f}\right|,\left|a_{3 i}^{f}\right| \lesssim 2000$ (with $i<3$ ).

We now proceed to examine several LFV observables sensitive to the couplings defined above, starting with purely leptonic processes.

\footnotetext{
${ }^{2}$ We factor out the gauge couplings in eq. (2.1) in such a way that the Wilson coefficients $c_{\gamma \gamma}$ and $c_{g g}$ become scale invariant at one-loop order [30].
} 

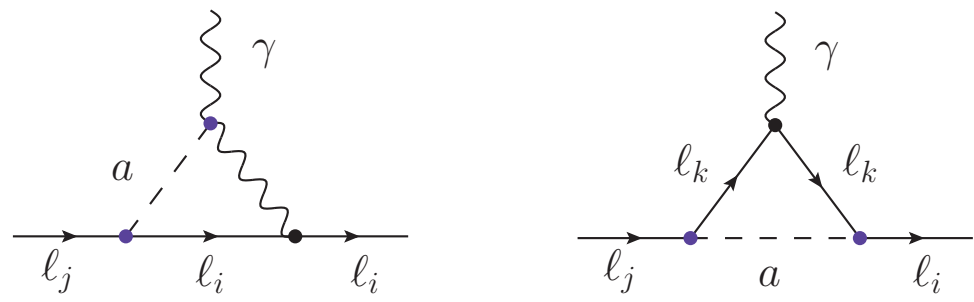

Figure 1. Diagrams illustrating the ALP contributions to $\ell_{j} \rightarrow \ell_{i} \gamma$ at linear (left panel) and quadratic (right panel) order in the Yukawa couplings.

\section{Purely leptonic processes}

The most promising decay channels in this category are the radiative decays $\ell_{j} \rightarrow \ell_{i} \gamma$, and the three-body decays $\ell_{j} \rightarrow \ell_{i} \ell_{k} \ell_{k}$ and $\ell_{j} \rightarrow \ell_{i} \gamma \gamma$ (with $i<j$ ). In the following, we provide the general expressions for each of these processes and discuss their potential in constraining ALP couplings.

\section{$3.1 \quad \ell_{j} \rightarrow \ell_{i} \gamma$}

The amplitude for the process $\ell_{j} \rightarrow \ell_{i} \gamma$ can be generically parameterized as

$$
i \mathcal{M}^{\mu}\left(\ell_{j} \rightarrow \ell_{i} \gamma\right)=i e \bar{u}_{i}(p-q) \Sigma_{i j}^{\mu}(0) u_{j}(p),
$$

where $p$ and $q$ denote the momentum of the heavy lepton and the photon, respectively. Since the photon is on-shell in this process, gauge invariance implies that the most general Dirac structure is given by

$$
\Sigma_{i j}^{\mu}(0)=\frac{i \sigma^{\mu \nu} q_{\nu}}{m_{\ell_{j}}}\left(\mathcal{F}_{2}^{i j}(0)+\mathcal{G}_{2}^{i j}(0) \gamma^{5}\right)
$$

where $\mathcal{F}_{2}^{i j}(0)$ and $\mathcal{G}_{2}^{i j}(0)$ are dimensionless form-factors. The above expression allows us to write the decay rate as

$$
\Gamma\left(\ell_{j} \rightarrow \ell_{i} \gamma\right)=\frac{m_{\ell_{j}}}{8 \pi} e^{2}\left(\left|\mathcal{F}_{2}^{i j}(0)\right|^{2}+\left|\mathcal{G}_{2}^{i j}(0)\right|^{2}\right),
$$

where $m_{\ell_{j}} \gg m_{\ell_{i}}$ has been used. The leading contributions to the dipole form-factors $\mathcal{F}_{2}^{i j}(0)$ and $\mathcal{G}_{2}^{i j}(0)$ come from the diagrams illustrated in figure 1 , which depend either linearly (left diagram) or quadratically (right diagram) on the lepton Yukawas, cf. eq. (2.2).

Linear diagrams, which mimic the so-called Barr-Zee contributions [50, 68], are typically dominant due to the logarithmic dependence on the ultraviolet cut-off. ${ }^{3}$ Their contribution to the dipole form factors reads

$$
\begin{aligned}
\mathcal{F}_{2}^{i j}(0)_{\text {lin. }} & =-e^{2} \frac{m_{\ell_{j}}^{2}}{8 \pi^{2} \Lambda^{2}} a_{i j}^{\ell} c_{\gamma \gamma} g_{\gamma}\left(x_{j}\right), \\
\mathcal{G}_{2}^{i j}(0)_{\text {lin. }} & =-e^{2} \frac{m_{\ell_{j}}^{2}}{8 \pi^{2} \Lambda^{2}} v_{i j}^{\ell} c_{\gamma \gamma} g_{\gamma}\left(x_{j}\right),
\end{aligned}
$$

\footnotetext{
${ }^{3}$ In ultraviolet complete models, the $c_{\gamma \gamma}$ arises only at loop-level, in such a way that this diagram would correspond to a two-loop contribution.
} 
where $x_{j}=m_{a}^{2} / m_{\ell_{j}}^{2}-i \eta\left(\right.$ with $\eta \rightarrow 0^{+}$) and $g_{\gamma}(x) \approx 2 \log \Lambda^{2} / m_{a}^{2}$. The complete loop function is reported in appendix A.1.

By contrast, the quadratic contributions are finite, once self-energies are included, and depend on the flavor of the lepton $\ell_{k}$ running in the loop. The contributions involving only one LFV coupling (i.e. with $k=i$ or $k=j$ ) $\operatorname{read}^{4}$

$$
\begin{aligned}
\mathcal{F}_{2}^{i j}(0)_{\text {quad. }} & =-\frac{m_{\ell_{j}}}{16 \pi^{2} \Lambda^{2}} a_{i j}^{\ell}\left[a_{j j}^{\ell} m_{\ell_{j}} g_{1}\left(x_{j}\right)+a_{i i}^{\ell} m_{\ell_{i}} g_{2}\left(x_{j}\right)\right], \\
\mathcal{G}_{2}^{i j}(0)_{\text {quad. }} & =-\frac{m_{\ell_{j}}}{16 \pi^{2} \Lambda^{2}} v_{i j}^{\ell}\left[a_{j j}^{\ell} m_{\ell_{j}} g_{1}\left(x_{j}\right)-a_{i i}^{\ell} m_{\ell_{i}} g_{2}\left(x_{j}\right)\right] .
\end{aligned}
$$

In the $\mu \rightarrow e \gamma$ case, there is an additional contribution from the $\tau$-loop exchange which is induced by a double LFV source. We find

$$
\begin{aligned}
& \mathcal{F}_{2}^{e \mu}(0)_{\text {quad. }}=-\frac{m_{\mu} m_{\tau}}{32 \pi^{2} \Lambda^{2}}\left(a_{e \tau}^{\ell} a_{\tau \mu}^{\ell}-v_{e \tau}^{\ell} v_{\tau \mu}^{\ell}\right) g_{3}\left(x_{\tau}\right), \\
& \mathcal{G}_{2}^{e \mu}(0)_{\text {quad. }}=-\frac{m_{\mu} m_{\tau}}{32 \pi^{2} \Lambda^{2}}\left(v_{e \tau}^{\ell} a_{\tau \mu}^{\ell}-a_{e \tau}^{\ell} v_{\tau \mu}^{\ell}\right) g_{3}\left(x_{\tau}\right),
\end{aligned}
$$

which show a $m_{\tau} / m_{\mu}$ enhancement compared to contributions involving a single LFV coupling. Similarly, $\tau \rightarrow \mu \gamma$ receives contributions from electron loops, which read

$$
\begin{aligned}
\mathcal{F}_{2}^{\mu \tau}(0)_{\text {quad }} & =-\frac{m_{\mu} m_{\tau}}{32 \pi^{2} \Lambda^{2}}\left(a_{\tau e}^{\ell} a_{\mu e}^{\ell}+v_{\tau e}^{\ell} v_{\mu e}^{\ell}\right) g_{4}\left(x_{\tau}\right), \\
\mathcal{G}_{2}^{\mu \tau}(0)_{\text {quad }} & =+\frac{m_{\mu} m_{\tau}}{32 \pi^{2} \Lambda^{2}}\left(a_{\tau e}^{\ell} v_{\mu e}^{\ell}+v_{\tau e}^{\ell} a_{\mu e}^{\ell}\right) g_{4}\left(x_{\tau}\right),
\end{aligned}
$$

while muon loops contribute to $\tau \rightarrow e \gamma$ as follows,

$$
\begin{aligned}
& \mathcal{F}_{2}^{e \tau}(0)_{\text {quad }}=-\frac{m_{\mu} m_{\tau}}{32 \pi^{2} \Lambda^{2}}\left(a_{\tau \mu}^{\ell} a_{e \mu}^{\ell}-v_{\tau \mu}^{\ell} v_{e \mu}^{\ell}\right) g_{4}\left(x_{\tau}\right), \\
& \mathcal{G}_{2}^{e \tau}(0)_{\text {quad }}=-\frac{m_{\mu} m_{\tau}}{32 \pi^{2} \Lambda^{2}}\left(a_{\tau \mu}^{\ell} v_{e \mu}^{\ell}-v_{\tau \mu}^{\ell} a_{e \mu}^{\ell}\right) g_{4}\left(x_{\tau}\right),
\end{aligned}
$$

with the loop functions $g_{i}(x)$ also collected in appendix A. We find full agreement with the results reported in ref. [49].

In summary, the most general contributions to $\ell_{j} \rightarrow \ell_{i} \gamma$ with a single LFV coupling are given by the sum of the linear and quadratic contributions, see eq. (3.4)-(3.7). Moreover, one should include the additional effects of eq. (3.8) and (3.9) in the $\mu \rightarrow e \gamma$ case, eq. (3.10) and (3.11) for $\tau \rightarrow \mu \gamma$, and finally eq. (3.12) and (3.13) for $\tau \rightarrow e \gamma$.

\section{$3.2 \quad \ell_{j} \rightarrow \ell_{i} \ell_{k} \ell_{k}$}

The processes $\ell_{j} \rightarrow \ell_{i} \ell_{k} \ell_{k}$ are described by the diagrams shown in figure 2 . The ALP can contribute both at tree-level (right panel) or at one loop, via the effective $\ell_{j} \rightarrow \ell_{i} \gamma^{*}$ vertex (left panel). Depending on the ALP mass, two different regimes arise: (i) for $m_{a}>m_{\ell_{j}}-m_{\ell_{i}}$ or $m_{a}<2 m_{\ell_{k}}$ the ALP is never produced on-shell, in such a way that there is a competition between tree and loop-level contributions, while (ii) for $2 m_{\ell_{k}}<m_{a}<m_{\ell_{j}}-m_{\ell_{i}}$

\footnotetext{
${ }^{4}$ For generality, we have kept the contributions from a light lepton running in the loop in the second terms of eq. (3.6) and (3.7). Nonetheless, these contributions turn out to be sub-dominant in most scenarios due to the suppression factor $m_{\ell_{i}} / m_{\ell_{j}} \ll 1$.
} 

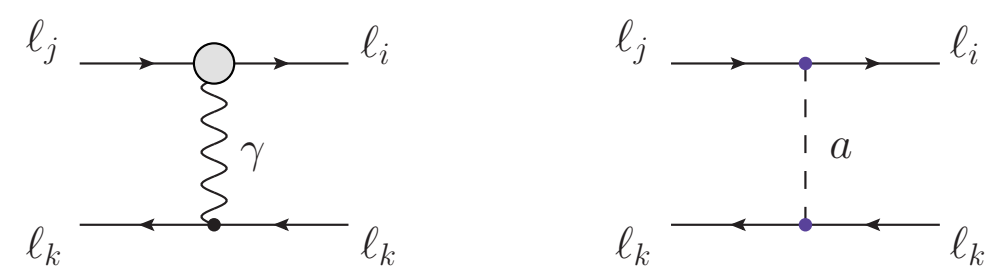

Figure 2. Diagrams illustrating the ALP contributions to $\ell_{j} \rightarrow \ell_{i} \ell_{k} \ell_{k}$ at loop-level (left panel) and tree-level (right panel). The gray blob in the photonic contribution represents the different loop contributions illustrated in figure 1.

the ALP can be produced on-shell, making the tree-level exchange dominant. In the following we provide the relevant expressions in both cases and discuss the phenomenological implications.

We start by parameterizing the general amplitude for the emission of an off-shell photon. In this case, eq. (3.1) should be replaced by

$$
i \mathcal{M}^{\mu}\left(\ell_{j} \rightarrow \ell_{i} \gamma^{*}\right)=i e \bar{u}_{i}(p-q) \Sigma_{i j}^{\mu}\left(q^{2}\right) u_{j}(p)
$$

with

$$
\Sigma_{i j}^{\mu}\left(q^{2}\right)=\gamma_{\nu}\left(\mathcal{F}_{1}^{i j}\left(q^{2}\right)+\mathcal{G}_{1}^{i j}\left(q^{2}\right) \gamma^{5}\right)\left(g^{\mu \nu}-\frac{q^{\mu} q^{\nu}}{q^{2}}\right)+\frac{i \sigma^{\mu \nu} q_{\nu}}{m_{\ell_{j}}}\left(\mathcal{F}_{2}^{i j}\left(q^{2}\right)+\mathcal{G}_{2}^{i j}\left(q^{2}\right) \gamma^{5}\right),
$$

where the $\mathcal{F}_{1,2}^{i j}\left(q^{2}\right)$ and $\mathcal{G}_{1,2}^{i j}\left(q^{2}\right)$ are form-factors depend on $q^{2}$ and on the masses of the particles running in the loops depicted in figure 1. The general expression for these functions, which are reported in appendix A.3, have been computed by independently using the packages FEYNCALC [69] and PACKAGE-X [70]. We verified that these expressions coincide with the results given in section 3.1 in the limit $q^{2} \rightarrow 0$. In particular, $\mathcal{F}_{1}^{i j}(0)=\mathcal{G}_{1}^{i j}(0)=0$, as expected by gauge invariance.

Even though the form-factors reported in appendix A.3 provide the most general description of the transition $\ell_{j} \rightarrow \ell_{i} \gamma^{*}$, it is convenient to derive simplified expressions which are valid for off-shell ALPs, i.e. for $m_{a}>m_{\ell_{j}}-m_{\ell_{i}}$, and which are more convenient for phenomenological analyses. In this case, $\mathcal{F}_{1}^{i j}$ and $\mathcal{G}_{1}^{i j}$ are well-approximated by a series around $q^{2}=0$,

$$
\begin{aligned}
& \mathcal{F}_{1}^{i j}\left(q^{2}\right)=q^{2} \dot{\mathcal{F}}_{1}^{i j}(0)+\mathcal{O}\left(q^{4}\right), \\
& \mathcal{G}_{1}^{i j}\left(q^{2}\right)=q^{2} \dot{\mathcal{G}}_{1}^{i j}(0)+\mathcal{O}\left(q^{4}\right),
\end{aligned}
$$

where $\dot{\Phi} \equiv \mathrm{d} \Phi / \mathrm{d} q^{2}$. Similarly, the dipole form-factors $\mathcal{F}_{2}^{i j}\left(q^{2}\right)$ and $\mathcal{G}_{2}^{i j}\left(q^{2}\right)$ are well described at leading order by setting $q^{2}=0$. The complete expressions for $\mathcal{F}_{2}^{i j}(0), \dot{\mathcal{F}}_{1}^{i j}(0), \mathcal{G}_{2}^{i j}(0)$ and $\dot{\mathcal{G}}_{1}^{i j}(0)$ are reported in appendix A.4.

The one-loop contributions computed above can now be combined with the tree-level one (see figure 2) to provide the general expression for $\Gamma\left(\ell_{j} \rightarrow \ell_{i} \ell_{k} \ell_{k}\right)$. For compactness, form factors are expanded around $q^{2}=0$, as described above. 
Off-shell decay rates. We first consider the off-shell scenario with $m_{a}>m_{\ell_{j}}-m_{\ell_{i}}$. The $\ell_{j} \rightarrow \ell_{i} \ell_{k} \ell_{k}$ decay rate in this regime can be decomposed in three pieces, namely (i) the photonic contribution, (ii) the ALP-mediated tree-level exchange and (iii) their interference, namely

$$
\Gamma\left(\ell_{j} \rightarrow \ell_{i} \ell_{k} \ell_{k}\right)=\Gamma\left(\ell_{j} \rightarrow \ell_{i} \gamma^{*} \rightarrow \ell_{i} \ell_{k} \ell_{k}\right)+\Gamma\left(\ell_{j} \rightarrow \ell_{i} a^{*} \rightarrow \ell_{i} \ell_{k} \ell_{k}\right)+\delta_{i k} \Gamma\left(\ell_{j} \rightarrow 3 \ell_{i}\right) \text { int. } .
$$

We compute each of these contributions by keeping the leading $q^{2}$-dependence in the oneloop form factors. For the photonic contribution, we find

$$
\begin{aligned}
\Gamma\left(\ell_{j} \rightarrow \ell_{i} \gamma^{*} \rightarrow \ell_{i} \ell_{k} \ell_{k}\right)=\frac{\alpha_{\mathrm{em}}^{2} m_{\ell_{j}}}{6 \pi}\left\{\left(\log \frac{m_{\ell_{j}}^{2}}{m_{\ell_{i}}^{2}}-3+\frac{\delta_{i k}}{4}\right)\left[\left|F_{2}^{i j}(0)\right|^{2}+\left|G_{2}^{i j}(0)\right|^{2}\right]\right. \\
\left.+\left(1+\frac{\delta_{i k}}{2}\right)\left[\frac{m_{\ell_{j}}^{4}}{4}\left(\left|\dot{F}_{1}^{i j}(0)\right|^{2}+\left|\dot{G}_{1}^{i j}(0)\right|^{2}\right)-m_{\ell_{j}}^{2} \operatorname{Re}\left(\dot{F}_{1}^{i j}(0) F_{2}^{i j}(0)^{*}-\dot{G}_{1}^{i j}(0) G_{2}^{i j}(0)^{*}\right)\right]\right\},
\end{aligned}
$$

where we have used $m_{\ell_{j}} \gg m_{\ell_{i}}$, finding agreement with the standard expressions available in the literature [71]. For the tree-level term, we obtain the following expression by neglecting the ALP width,

$$
\Gamma\left(\ell_{j} \rightarrow \ell_{i} a^{*} \rightarrow \ell_{i} \ell_{k} \ell_{k}\right)=\frac{\left|a_{k k}^{\ell}\right|^{2}\left|s_{i j}^{\ell}\right|^{2}}{32 \pi^{3}} \frac{m_{\ell_{j}}^{3} m_{\ell_{k}}^{2}}{\Lambda^{4}} \varphi_{0}^{i k}\left(x_{j}\right)
$$

where $x_{j}=m_{a}^{2} / m_{\ell_{j}}^{2}$, as before, and the phase-space function $\varphi_{0}^{i k}(x)$ is reported in appendix A.2. In the limit of large ALP masses, this function satisfies $\varphi_{0}^{i k}(x) \propto 1 / x^{2}+$ $\mathcal{O}\left(1 / x^{3}\right)$, in agreement with the decoupling limit. Similarly, we computed the interference of both contributions, which is given by

$$
\begin{aligned}
\Gamma\left(\ell_{j} \rightarrow 3 \ell_{i}\right)_{\text {int. }}=\frac{\alpha_{\mathrm{em}}}{96 \pi^{2}} \frac{m_{\ell_{j}}^{2} m_{\ell_{i}}}{\Lambda^{2}}\{ & -2 \varphi_{1}\left(x_{j}\right) \operatorname{Re}\left[a_{11}^{\ell}\left(a_{21}^{\ell} F_{2}(0)^{*}-v_{21}^{\ell} G_{2}(0)^{*}\right)\right], \\
& \left.+m_{\ell_{j}}^{2} \varphi_{2}\left(x_{j}\right) \operatorname{Re}\left[a_{11}^{\ell}\left(a_{21}^{\ell} \dot{F}_{1}(0)^{*}+v_{21}^{\ell} \dot{G}_{1}(0)^{*}\right)\right]\right\},
\end{aligned}
$$

where the phase-space functions satisfy $\varphi_{1,2}(x)=1 / x+\mathcal{O}\left(1 / x^{2}\right)$, with the complete functions collected in appendix A.2. Note that the interference between tree and loop-level contributions vanishes identically for $k \neq i$. The phenomenological implication of these expressions are discussed in section 3.5.

In the off-shell scenario with $m_{a}<2 m_{\ell_{k}}$, eq. (3.18) remains the same, while eq. (3.19) and (3.20) should be reevaluated with the appropriate phase-space integration. In this case, it is more difficult to provide a compact analytical expression as there are more mass scales involved. In the phenomenological analysis, we integrate the form factors reported in appendix A.3 numerically. We have also checked that the form factor expansion around $q^{2}=0$ remains a reasonable approximation 
On-shell decay rates. The above expressions can be simplified in the case where the ALP is produced on-shell, i.e. for $2 m_{\ell_{k}}<m_{a}<m_{\ell_{j}}-m_{\ell_{i}}$. In this case, the interference term in eq. (3.20) becomes negligible, while the photonic contribution remains identical to eq. (3.18). On the other hand, the tree-level ALP exchange can be described in the narrow-width approximation,

$$
\Gamma\left(\ell_{j} \rightarrow \ell_{i} a \rightarrow \ell_{i} \ell_{k} \ell_{k}\right) \approx \Gamma\left(\ell_{j} \rightarrow \ell_{i} a\right) \mathcal{B}\left(a \rightarrow \ell_{k} \ell_{k}\right),
$$

with

$$
\Gamma\left(\ell_{j} \rightarrow \ell_{i} a\right)=\frac{m_{\ell_{j}}^{3}}{16 \pi}\left(1-\frac{m_{a}^{2}}{m_{\ell_{j}}^{2}}\right)^{2} \frac{\left|s_{i j}^{\ell}\right|^{2}}{\Lambda^{2}},
$$

where we have used that $m_{\ell_{j}} \gg m_{\ell_{i}}$, and

$$
\mathcal{B}\left(a \rightarrow \ell_{k}^{-} \ell_{k}^{+}\right)=\tau_{a} \frac{\left|a_{k k}^{\ell}\right|^{2}}{\Lambda^{2}} \frac{m_{a} m_{\ell_{k}}^{2}}{2 \pi} \sqrt{1-\frac{4 m_{\ell_{k}}^{2}}{m_{a}^{2}}},
$$

where $\tau_{a}$ denotes the ALP lifetime.

To assess the limits on ALP couplings in the on-shell regime, one should estimate the ALP flight distance and verify that it decays inside the detector, as shall be discussed next. The ALP boosted decay length in the lab frame is given by

$$
l_{a}=\frac{c\left|p_{a}\right|}{m_{a} \Gamma_{a}}
$$

where $p_{a}$ denotes the ALP momentum in the lab frame. By assuming that $\ell_{j}$ decays at rest, as in the case of $\mu \rightarrow e$ experiments such as MEG [72] or Mu3e [44], $\left|p_{a}\right|$ reads

$$
\left|p_{a}\right|=\frac{\lambda^{1 / 2}\left(m_{a}, m_{\ell_{j}}, m_{\ell_{i}}\right)}{2 m_{\ell_{j}}}
$$

with $\lambda(a, b, c) \equiv\left(a^{2}-(b-c)^{2}\right)\left(a^{2}-(b+c)^{2}\right)$. In our numerical analysis, we will naively impose the relaxed bound that $\ell_{a}$ is not larger than $\approx 1 \mathrm{~m}$ in order for the ALP decay to be considered prompt. A more refined analysis can be performed in experimental searches, by using the displaced vertex as a tool to set even more stringent limits than searches based on prompt decays $[47,48] .{ }^{5}$ Another limitation of our analysis is the reinterpretation of $\tau \rightarrow e$ and $\tau \rightarrow \mu$ limits. Indeed, since $\tau$ 's are not produced at rest in current experiments, eq. (3.25) does not apply in this case. The correct assessment of $\tau$ LFV limits in the resonant region would require a dedicated experimental study, as already suggested in refs. $[47,48]$

\section{$3.3 \quad \ell_{j} \rightarrow \ell_{i} \gamma \gamma$}

The next purely leptonic decay mode we discuss is $\ell_{j} \rightarrow \ell_{i} \gamma \gamma$. We focus on the tree-level contribution illustrated in figure 3, which is the dominant one for light ALPs. Once again, we separate the off-shell region, $m_{a}>m_{\ell_{j}}-m_{\ell_{i}}$, from the on-shell one, $m_{a}<m_{\ell_{j}}-m_{\ell_{i}}$.

\footnotetext{
${ }^{5}$ Similar displaced-vertex searches have been recently performed by LHCb in the decays $B \rightarrow K^{(*)} a \rightarrow$ $K^{(*)} \mu \mu[73,74]$, which provide some of the most stringent limits on GeV ALPs with couplings to both quarks and leptons $[39,40]$.
} 


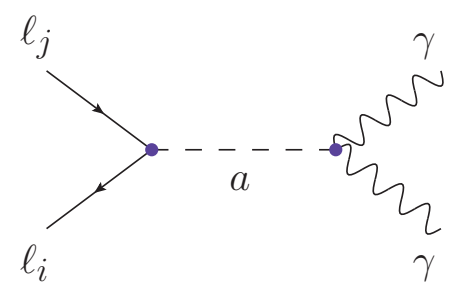

Figure 3. Dominant contribution to $\ell_{j} \rightarrow \ell_{i} \gamma \gamma$ for light ALPs, i.e. $m_{a} \lesssim m_{\ell_{j}}$.

Off-shell decay rates. The general expression for the branching ratio of $\ell_{j} \rightarrow \ell_{i} \gamma \gamma$ in the off-shell regime $\left(m_{a}>m_{\ell_{j}}\right)$ is given by

$$
\mathcal{B}\left(\ell_{j} \rightarrow \ell_{i} \gamma \gamma\right)=\tau_{\ell_{i}} \frac{e^{4} c_{\gamma \gamma}^{2}\left|s_{i j}^{\ell}\right|^{2}}{\Lambda^{4}} \frac{m_{\ell_{i}}^{5}}{192 \pi^{3}} \varphi(x),
$$

where $x=m_{a}^{2} / m_{\ell_{j}}^{2}$ and the loop-function $\varphi(x)$ reads

$$
\varphi(x)=1+12 x(x-1)+6 x\left(2 x^{2}-3 x+1\right) \log \frac{x-1}{x},
$$

with $\varphi(x)=1 /\left(10 x^{2}\right)+\mathcal{O}\left(1 / x^{3}\right)$ as $x \gg 1$.

On-shell decay rates. In the on-shell regime, the branching ratio of $\ell_{j} \rightarrow \ell_{i} \gamma \gamma$ can be obtained exploiting the narrow-width approximation, leading to the following result

$$
\mathcal{B}\left(\ell_{j} \rightarrow \ell_{i} \gamma \gamma\right) \approx \mathcal{B}\left(\ell_{j} \rightarrow \ell_{i} a\right) \mathcal{B}(a \rightarrow \gamma \gamma)
$$

where

$$
\mathcal{B}(a \rightarrow \gamma \gamma)=\tau_{a} \frac{e^{4}\left|c_{\gamma \gamma}\right|^{2}}{\Lambda^{2}} \frac{m_{a}^{3}}{4 \pi},
$$

and the leptonic decay rate is given in eq. (3.22). As discussed in section 3.5, given the present experimental constraints, the process $\ell_{j} \rightarrow \ell_{i} \gamma \gamma$ turns out to be effective in limiting the ALP parameter space only in the on-shell regime.

\section{$3.4 \mu^{-} \rightarrow e^{-}$conversion in nuclei}

We now discuss $\mu^{-} \rightarrow e^{-}$conversion in nuclei, which will become one of the most sensitive LFV probes in the coming years (cf. refs. $[75,76]$ for an overview of experimental prospects). We consider this observable to be a purely leptonic probe, since ALP couplings to quarks and gluons do not induce coherent contributions to the conversion rate, being therefore negligible [71]. The only relevant contributions are then the ones stemming from the (offshell) photon penguins computed in section 3.2. ${ }^{6}$

The conversion branching fraction is defined as the ratio of the $\mu \rightarrow e$ conversion rate over the nuclear capture one. Following ref. [71], we have

$$
\mathcal{B}\left(\mu^{-} N \rightarrow e^{-} N\right)=\frac{8 \alpha^{5} m_{\mu} Z_{\text {eff }}^{4} Z F_{p}^{2}}{\Gamma_{\text {capt }}} \xi^{2},
$$

\footnotetext{
${ }^{6}$ See refs. $[77,78]$ for an estimation of spin-dependent contributions to these processes arising from axial-vector and tensor operators.
} 
where $Z_{\text {eff }}$ is the effective atomic charge, $F_{p}$ parameterizes the nuclear matrix element and $\Gamma_{\text {capt }}$ stands for the total muon capture rate. The factor $\xi^{2}$ accounts for the photonexchange contributions as follows

$$
\xi^{2}=\left|\mathcal{F}_{1}^{\mu e}\left(-m_{\mu}^{2}\right)+\mathcal{F}_{2}^{\mu e}\left(-m_{\mu}^{2}\right)\right|^{2}+\left|\mathcal{G}_{1}^{\mu e}\left(-m_{\mu}^{2}\right)-\mathcal{G}_{2}^{\mu e}\left(-m_{\mu}^{2}\right)\right|^{2},
$$

where $\mathcal{F}_{1,2}\left(-m_{\mu}^{2}\right)$ and $\mathcal{G}_{1,2}\left(-m_{\mu}^{2}\right)$ are the $\mu \rightarrow e \gamma^{*}$ form factors evaluated at $q^{2}=-m_{\mu}^{2}$, cf. eq. (3.15) and appendix A.3. For $m_{a}>m_{\mu}$, evaluating the form-factors at $q^{2}=0$ is a very good approximation. In particular, considering only one flavor-violating coupling and neglecting the electron mass, this expression simplifies to

$$
\xi^{2} \propto\left|v_{\mu e}^{\ell}\right|^{2}+\left|a_{\mu e}^{\ell}\right|^{2}=\left|s_{\mu e}^{\ell}\right|^{2}
$$

as in the LFV quantities discussed above. In the specific case of gold $\left({ }_{79}^{197} \mathrm{Au}\right)$ and aluminum $\left({ }_{13}^{27} \mathrm{Al}\right)$ atoms, the necessary inputs are given respectively by $[79,80]$,

$$
\begin{array}{llll}
Z=79, & Z_{\text {eff }}=33.5, & F_{p}=0.16, & \Gamma_{\text {capt }}=8.59868 \cdot 10^{-18} \mathrm{GeV}, \\
Z=13, & Z_{\text {eff }}=11.5, & F_{p}=0.64, & \Gamma_{\text {capt }}=4.64079 \times 10^{-19} \mathrm{GeV},
\end{array}
$$

which can be replaced in eq. (3.30) to give

$$
\mathcal{B}^{\text {th }}\left(\mu^{-} \mathrm{Au} \rightarrow e^{-} \mathrm{Au}\right) \approx 5.2 \times 10^{12} \xi^{2}, \quad \mathcal{B}^{\text {th }}\left(\mu^{-} \mathrm{Al} \rightarrow e^{-} \mathrm{Al}\right) \approx 3.52 \times 10^{12} \xi^{2} .
$$

Currently, the most stringent limit is $\mathcal{B}\left(\mu^{-} \mathrm{Au} \rightarrow e^{-} \mathrm{Au}\right)<7 \times 10^{-13}$, obtained by the Sindrum-II collaboration [81]. In the near future, the Mu2e experiment at Fermilab [44] and Comet [46] at J-PARC aim to improve the experimental sensitivity to $\mathcal{O}\left(10^{-17}\right)$ by using aluminum atoms, cf. table 1 .

\subsection{Numerical results and discussion}

In this section we discuss the phenomenological implications of the results obtained above. Our main goal is to explore the complementarity of the different leptonic probes and their experimental prospects at current/future experimental facilities.

To derive constraints from existing data, we focus on a benchmark scenario defined by lepton-flavor universal couplings to leptons $\left(a^{\ell} \equiv a_{i i}^{\ell}\right)$, with the other flavor-conserving couplings vanishing at tree-level. In this case, the effective $a \gamma \gamma$ coupling is generated by the (irreducible) one-loop contributions from leptonic couplings [30],

$$
c_{\gamma \gamma}^{\text {loop }}=\frac{a^{\ell}}{8 \pi^{2}} \sum_{i=e, \mu, \tau} B_{1}\left(\tau_{i}\right)
$$

where $\tau_{i}=4 m_{i}^{2} / m_{a}^{2}-i \eta$ and the loop-function is given by

$$
B_{1}(\tau)=1-\tau f^{2}(\tau) \quad \text { with } \quad f(\tau)= \begin{cases}\arcsin \frac{1}{\sqrt{\tau}}, & \tau \geq 1 \\ \frac{\pi}{2}+\frac{i}{2} \log \frac{1+\sqrt{1-\tau}}{1-\sqrt{1-\tau}}, & \tau<1\end{cases}
$$




\begin{tabular}{|c|ccc|}
\hline Decay mode & Exp. limit & Future prospects & Ref. \\
\hline$\mu \rightarrow e \gamma$ & $4.2 \times 10^{-13}$ & $\approx 6 \times 10^{-14}$ & {$[82]$} \\
$\mu \rightarrow 3 e$ & $1.0 \times 10^{-12}$ & $\approx 10^{-16}$ & {$[82]$} \\
$\mu \rightarrow e \gamma \gamma$ & $7.2 \times 10^{-11}$ & - & {$[82]$} \\
$\mu \rightarrow e+\mathrm{inv}$ & $\approx 10^{-5}$ & - & {$[83]$} \\
$\mu^{-} \mathrm{Ti} \rightarrow e^{-} \mathrm{Ti}$ & $4.3 \times 10^{-12}$ & - & {$[84]$} \\
$\mu^{-} \mathrm{Au} \rightarrow e^{-} \mathrm{Au}$ & $7 \times 10^{-13}$ & $\approx 10^{-17}$ & {$[81]$} \\
$\mu^{-} \mathrm{Al} \rightarrow e^{-} \mathrm{Al}$ & - & $\approx 10^{-17}$ & {$[44,46]$} \\
\hline$\tau \rightarrow e \gamma$ & $3.3 \times 10^{-8}$ & $\approx 3 \times 10^{-9}$ & {$[82]$} \\
$\tau \rightarrow 3 e$ & $2.7 \times 10^{-8}$ & $\approx 5 \times 10^{-10}$ & {$[82]$} \\
$\tau \rightarrow e \mu^{+} \mu^{-}$ & $1.7 \times 10^{-8}$ & $\approx 6 \times 10^{-10}$ & {$[82]$} \\
$\tau \rightarrow e+\mathrm{inv}$ & $\approx 5 \times 10^{-3}$ & - & {$[85]$} \\
\hline$\tau \rightarrow \mu \gamma$ & $4.4 \times 10^{-8}$ & $\approx 10^{-9}$ & {$[82]$} \\
$\tau \rightarrow 3 \mu$ & $2.1 \times 10^{-8}$ & $\approx 4 \times 10^{-10}$ & {$[82]$} \\
$\tau \rightarrow \mu e^{+} e^{-}$ & $1.8 \times 10^{-8}$ & $\approx 3 \times 10^{-10}$ & {$[82]$} \\
$\tau \rightarrow \mu+\mathrm{inv}$ & $\approx 5 \times 10^{-3}$ & - & {$[85]$} \\
\hline
\end{tabular}

Table 1. Most relevant experimental limits on purely leptonic LFV processes and future prospects for MEG-II [86], Mu2E [44], Mu3E [45], COMET [46] and Belle-II [43].

which behaves as $B_{1}(\tau) \approx 1 / \sqrt{\tau}$ in the $\tau \rightarrow \infty$ asymptotic limit, in such a way that only fermions lighter than $m_{a}$ contribute significantly to eq. (3.36). To derive the relevant constraints to this scenario, it is crucial to determine the ALP total width, $\Gamma_{a}$, and its flight distance in a given experiment $[47,48]$, as discussed above. The constraints on $\left|a_{\mu e}^{\ell}\right|$, $\left|a_{\tau e}^{\ell}\right|$ and $\left|a_{\tau \mu}^{\ell}\right|$ are shown in figure 4 , as a function of the ALP mass $m_{a}$, by setting the flavor-conserving coupling to $a^{\ell}=1$. Several comments are in order:

- Out of the whole set of $\mu \rightarrow e$ processes, $\mathcal{B}(\mu \rightarrow 3 e)$ and $\mathcal{B}(\mu \rightarrow e \gamma \gamma)$ impose the most stringent bounds for $20 \mathrm{MeV}<m_{a} \lesssim 100 \mathrm{MeV}$ due to the resonant-enhancement of the branching fractions. The upper bound corresponds to the kinematical threshold of on-shell ALP production, i.e. $m_{a}<m_{\mu}-m_{e}$, while the lower bound $m_{a} \gtrsim 20 \mathrm{MeV}$ comes from the requirement that the ALP decays inside the detector, i.e. $\ell_{a} \lesssim 1 \mathrm{~m}$, cf. eq. (3.24).

- For $m_{a} \lesssim 20 \mathrm{MeV}$, the ALP decays outside of the detector, in such a way that $\mu \rightarrow e a$ is indistinguishable from $\mu \rightarrow e+$ inv., from which we obtain the most stringent constraint in this mass range. In principle, these limits could be improved if dedicated experimental searches for displaced vertices are performed [47, 48].

- For $m_{a}>m_{\mu}$, the process $\mathcal{B}(\mu \rightarrow 3 e)$ is dominated by the loop exchange of ALPs. Indeed, while tree-level effects decouple with inverse powers of $m_{a}$, loop-induced 

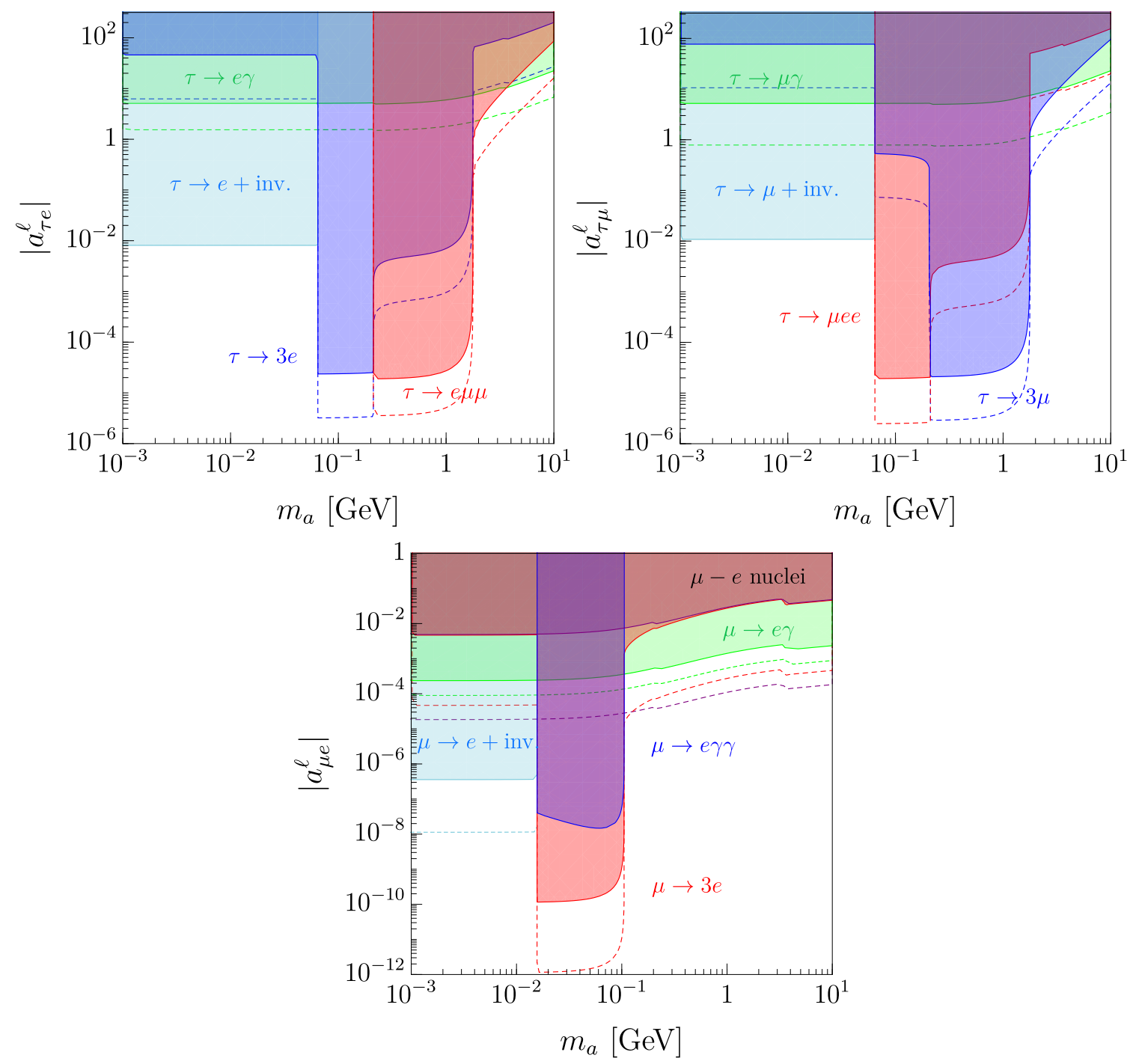

Figure 4. Constraints on $\left|s_{\mu e}^{\ell}\right|$ (lower panel), $\left|s_{\tau e}^{\ell}\right|$ (upper left panel) and $\left|s_{\tau \mu}^{\ell}\right|$ (upper right panel) as a function of the ALP mass derived from the experimental bounds listed in table 1. Dashed lines correspond to future experimental prospects. We consider a benchmark scenario where $a_{i i}^{\ell}=1$ are the only flavor-conserving tree-level couplings, while $c_{\gamma \gamma}$ is induced at one-loop level, cf. eq. (3.36). The other couplings are neglected in our analysis.

contributions exhibit a smoother logarithmic dependence. For this reason, $\mu \rightarrow e \gamma$ is currently the most constraining process for these masses. Currently, $\mu \rightarrow e$ conversion in nuclei is less sensitive to ALP couplings, since $\mathcal{B}(\mu N \rightarrow e N) \approx \mathcal{B}(\mu \rightarrow 3 e) \approx$ $10^{-2} \times \mathcal{B}(\mu \rightarrow e \gamma)$. This is expected to change in the future thanks to the Mu2E [44] and COMET [46] experiments, which will improve the present sensitivity by orders of magnitude, cf. table 1.

- Bounds on $\tau \rightarrow \mu$ and $\tau \rightarrow e$ transitions share similar features to the ones already outlined above for the $\mu \rightarrow e$ transition. An important difference stems from the 

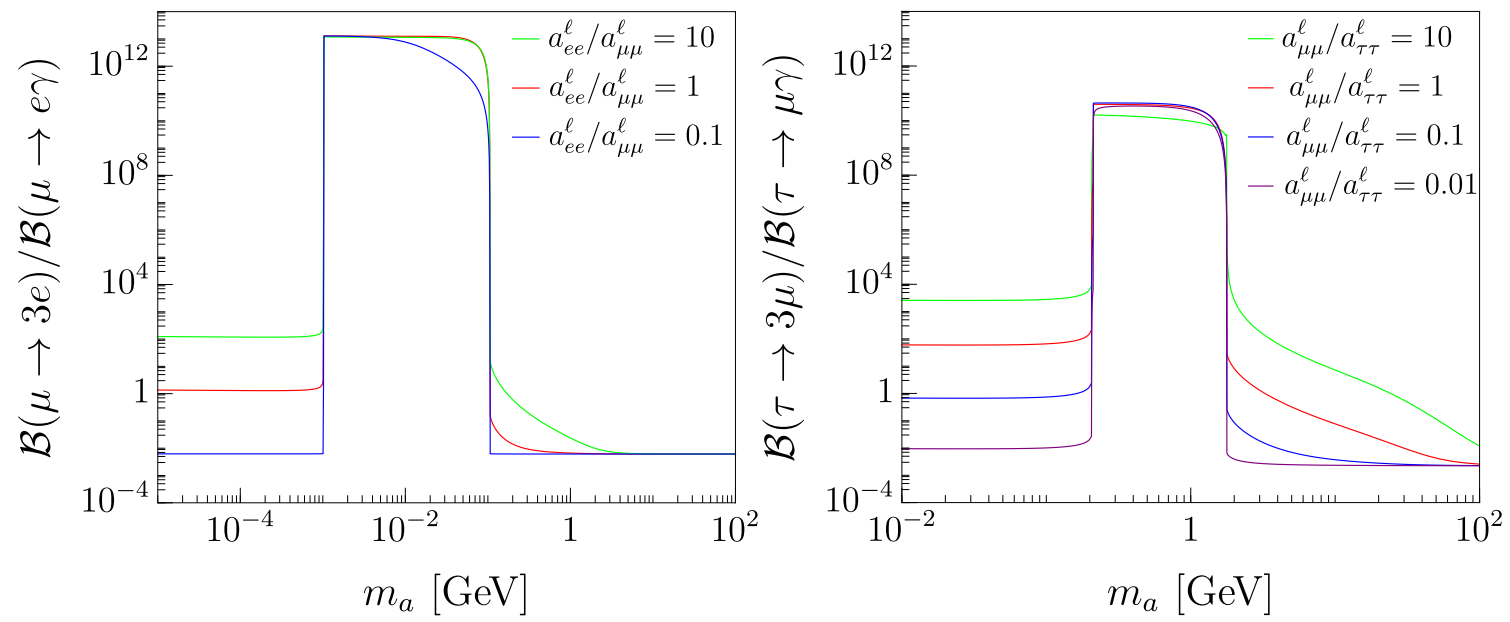

Figure 5. $\mathcal{B}(\mu \rightarrow 3 e) / \mathcal{B}(\mu \rightarrow e \gamma)$ and $\mathcal{B}(\tau \rightarrow 3 \mu) / \mathcal{B}(\tau \rightarrow \mu \gamma)$ as a function of $m_{a}$ for the benchmark ratios $a^{\ell} \equiv a_{i i}^{\ell}$ specified in the plot. The coupling $c_{\gamma \gamma}$ is assumed to be induced at one-loop. See the text for more details.

interplay between constraints from ALP on-shell production and the requirement that the ALP decays inside the detector. In particular, the three body decays $\tau \rightarrow 3 \mu$ and $\tau \rightarrow e \mu \mu$ set the most stringent bounds in the ranges $2 m_{\mu}<m_{a}<m_{\tau}-m_{\mu}$ and $2 m_{\mu}<m_{a}<m_{\tau}-m_{e}$, respectively, which correspond precisely to the kinematical thresholds for on-shell ALP production. On the other hand, $\tau \rightarrow \mu e e$ and $\tau \rightarrow 3 e$ are the most sensitive processes in the region $7 \mathrm{MeV} \lesssim m_{a}<2 m_{\mu}$, where the lower limit stems from the ALP lifetime constraint. Note, in particular, that our flavor ansatz is such that the relation $\mathcal{B}(\tau \rightarrow 3 \mu) / \mathcal{B}(\tau \rightarrow \mu e e)=\mathcal{B}(\tau \rightarrow e \mu \mu) / \mathcal{B}(\tau \rightarrow 3 e)=m_{\mu}^{2} / m_{e}^{2}$ holds exactly in the region where these observables are resonantly enhanced.

Let us now analyze the interplay between the different contributions to $\ell_{j} \rightarrow \ell_{i} \ell_{k} \ell_{k}$ computed in section 3.2 and the correlations among LFV processes. From the above discussion, it is clear that $\ell_{j} \rightarrow \ell_{i} \ell_{k} \ell_{k}$ is the dominant decay mode if the ALP can be produced on-shell, i.e. for $2 m_{\ell_{i}}<m_{a}<m_{\ell_{j}}-m_{\ell_{i}}$. On the other hand, this comparison is less evident below and above the resonant mass interval, as the interplay of the tree and loop-level contributions to $\ell_{j} \rightarrow 3 \ell_{i}$ becomes non-trivial. To better illustrate this feature, in figure 5 we plot $\mathcal{B}\left(\ell_{j} \rightarrow 3 \ell_{i}\right) / \mathcal{B}\left(\ell_{j} \rightarrow \ell_{i} \gamma\right)$ as a function of $m_{a}$ for $\mu \rightarrow e$ (left panel) and $\tau \rightarrow \mu$ (right panel) transitions. In this plot, the ALP couplings are set to $c_{\gamma \gamma}=1 /\left(16 \pi^{2}\right)$ and $a_{j j}^{\ell}=1$, with $a_{i i}^{\ell} / a_{j j}^{\ell}$ fixed to a few representative values. The LFV coupling $a_{j i}^{\ell}$ is not specified as it cancels in the ratios we are interested in, while the other couplings are neglected.

From figure 5 we see that for $m_{a}>m_{\ell_{j}}$ the tree-level contribution to $\ell_{j} \rightarrow 3 \ell_{i}$ decouples as $1 / m_{a}^{4}$, becoming subdominant compared to the loop-induced photon-penguin contributions, which have a milder logarithmic dependence on $m_{a}$. We find that tree-level contributions to $\mathcal{B}(\mu \rightarrow 3 e)$ can be neglected for $m_{a} \gtrsim 1 \mathrm{GeV}$, while masses as large as $m_{a} \approx 100 \mathrm{GeV}$ are needed for $\tau \rightarrow 3 \mu$ (see also figure 4). In both cases, for sufficiently large $m_{a}$ values, $\mathcal{B}\left(\ell \rightarrow 3 \ell_{i}\right) / \mathcal{B}\left(\ell_{j} \rightarrow \ell_{i} \gamma\right)$ approaches the prediction of dipole form-factor 
dominance,

$$
\frac{\mathcal{B}\left(\ell_{j} \rightarrow 3 \ell_{i}\right)}{\mathcal{B}\left(\ell_{j} \rightarrow \ell_{i} \gamma\right)} \approx \frac{\alpha_{\mathrm{em}}}{3 \pi}\left(\log \frac{m_{\ell_{j}}^{2}}{m_{\ell_{i}}^{2}}-\frac{11}{4}\right),
$$

which yields $\approx 6 \times 10^{-3}, \approx 10^{-2}$ and $\approx 2 \times 10^{-3}$ for the $\mu \rightarrow e, \tau \rightarrow e$ and $\tau \rightarrow \mu$ transitions, respectively. In principle, this relation can be broken by contributions to $\ell_{j} \rightarrow 3 \ell_{i}$ stemming from the anapole form-factors $\mathcal{F}_{1}\left(q^{2}\right)$ and $\mathcal{G}_{1}\left(q^{2}\right)$. Nonetheless, in most cases these contributions are sub-dominant with respect to the tree-level ones or the ones originating from dipoles. This can be understood from the ultraviolet logarithmic enhancement of the Barr-Zee dipole form-factor in eq. (3.4) and (3.5), and/or the kinematical logarithmic enhancement of eq. (3.18). Lastly, we also comment on the case where $m_{a}<2 m_{\ell_{i}}$. For these values of $m_{a}$, the tree-level contribution dominates for $\left|a_{i i}^{\ell}\right| /\left|a_{i j}^{\ell}\right| \lesssim 1$, as depicted in figure 5. Loop-induced contributions can become dominant only for large values of $a_{i i}^{\ell}$ compared to $a_{j j}^{\ell}$ and $c_{\gamma \gamma}$.

Another peculiar signature of ALP-induced LFV arises in the correlation between $\mu+N \rightarrow e+N$ and $\mu \rightarrow e \gamma$. If the effects from dipole form-factors are dominant, as predicted in many NP scenarios, the following prediction holds

$$
\frac{\mathcal{B}\left(\mu^{-}+\mathrm{Au} \rightarrow e^{-} \mathrm{Au}\right)}{\mathcal{B}(\mu \rightarrow e \gamma)} \approx 4 \times 10^{-3} .
$$

To investigate possible departures from this relation, we plot the same ratio in figure 6 for universal ALP couplings $a_{i i}^{\ell} \equiv a^{\ell}$ and various values of $c_{\gamma \gamma}$. For large values of $c_{\gamma \gamma}$ (red line), we find that the dipole effects driven by the Barr-Zee contributions dominate for any values of $m_{a}$, reproducing the results of eq. (3.39). For $c_{\gamma \gamma}= \pm 1 / 16 \pi^{2}$, the above relation is broken by the anapole contributions for small values of $m_{a}$. Instead, for large $m_{a}$, the Barr-Zee effects dominate due to their logarithmic sensitivity to $m_{a}$, cf. eq. (3.4), and eq. (3.39) holds. Finally, for $c_{\gamma \gamma}=0$, anapole and dipole effects have a comparable size for any value of $m_{a}$ yielding a significant departure from eq. (3.39).

Before moving into the discussion of processes involving hadrons, we turn our attention to the anomalous magnetic moment of leptons $(g-2)_{\ell}$ which are tightly related to LFV processes, as we are going to see in the following.

\subsection{On the $(g-2)_{e}$ and $(g-2)_{\mu}$ anomalies}

The anomalous magnetic moment of leptons, $a_{\ell}=(g-2)_{\ell} / 2$, provides one of the most accurate tests of the SM validity. The longstanding discrepancy between the experimental value and the SM prediction, $\Delta a_{\mu}=a_{\mu}^{\exp }-a_{\mu}^{\mathrm{SM}}=(27.1 \pm 7.3) \times 10^{-10}[51-53]$, at the level of $\approx 3.6 \sigma$, received increased attention recently due to the anticipated new experimental results by the Muon $g-2$ collaboration at Fermilab [54]. Furthermore, the recent measurement of the fine-structure constant $\alpha_{\mathrm{em}}$ in atomic physics experiments [63] allow us to concretely use for the first time the electron $g-2$ as a NP probe [55]. Surprisingly, the reevaluation of $\Delta a_{e}$ employing the latest value of $\alpha_{\mathrm{em}}$ shows a mild discrepancy $\Delta a_{e}=(-87 \pm 36) \times 10^{-14}[64,65]$, at the level of $2.4 \sigma$. This value not only has a different sign than $\Delta a_{\mu}$, it also shows a departure from the expectations derived by considering the "naive scaling", i.e. the assumption that $\Delta a_{\ell}$ scales as $m_{\ell}^{2}$, which is valid for a large class of NP models [55]. 


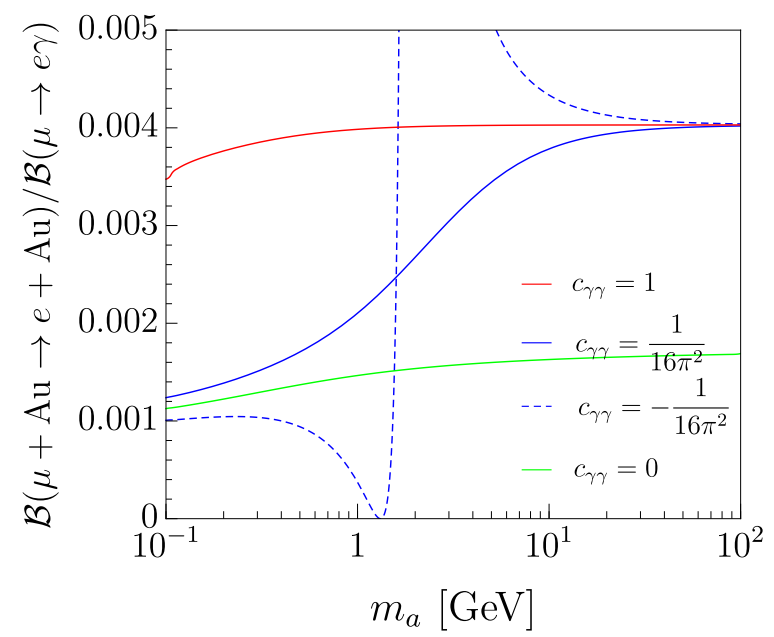

Figure 6. $\mathcal{B}(\mu+\mathrm{Au} \rightarrow e+\mathrm{Au}) / \mathcal{B}(\mu \rightarrow e \gamma)$ is plotted as a function of $m_{a}$ for $a^{\ell} \equiv a_{i i}^{\ell}$ universal and various values of the coupling $c_{\gamma \gamma}$. We assume that $a_{\mu e}^{\ell}$ is the only non-vanishing LFV coupling.

Light ALPs are promising candidates to accommodate both $g-2$ anomalies since they can contribute in different ways to $(g-2)_{\ell}$, with $\ell=e, \mu, \tau$, breaking the "naive scaling" expectations. The leading ALP contributions are given by the one-loop diagrams shown in figure 1 (with $i=j$ ), as well as by the two-loop light-by-light and vacuum polarization diagrams, which are entirely induced by the $a \gamma \gamma$ coupling [50]. The expression for $a_{\ell_{i}}=\mathcal{F}_{2}^{i i}(0)$ can then be written as

$$
\Delta a_{\ell_{i}}=\left(\Delta a_{\ell_{i}}\right)_{\mathrm{LFC}}+\left(\Delta a_{\ell_{i}}\right)_{\mathrm{LFV}},
$$

where the two contributions derive from lepton flavor-conserving (LFC) and LFV couplings, respectively. Indeed, although $a_{\ell_{i}}$ are flavor-conserving observables, in presence of LFV couplings they can receive contributions from loops involving a lepton of different flavor, cf. figure 1 with $i=j \neq k$. The LFC expression from Yukawa interactions is universal and reads

$$
\left(\Delta a_{\ell_{i}}\right)_{\mathrm{LFC}}=-\frac{m_{\ell_{i}}^{2}}{16 \pi^{2} \Lambda^{2}}\left[64 \pi \alpha_{\mathrm{em}} c_{\gamma \gamma} a_{i i}^{\ell}\left(\log \frac{\Lambda^{2}}{m_{\ell_{i}}^{2}}-h_{2}\left(x_{i}\right)\right)+4\left|a_{i i}^{\ell}\right|^{2} h_{1}\left(x_{i}\right)\right] .
$$

Two-loop contributions to $\left(\Delta a_{\ell_{i}}\right)_{\mathrm{LFC}}$ induced by $a \gamma \gamma$ are almost negligible for the ALP couplings we consider [50]. The LFV contributions are different for each lepton flavor and they can be expressed as

$$
\begin{aligned}
& \left(\Delta a_{e}\right)_{\mathrm{LFV}}=\frac{m_{e}^{2}}{16 \pi^{2} \Lambda^{2}}\left[\frac{m_{\mu}}{m_{e}}\left(\left|v_{e \mu}^{\ell}\right|^{2}-\left|a_{e \mu}^{\ell}\right|^{2}\right) g_{3}\left(x_{\mu}\right)+\frac{m_{\tau}}{m_{e}}\left(\left|v_{e \tau}^{\ell}\right|^{2}-\left|a_{e \tau}^{\ell}\right|^{2}\right) g_{3}\left(x_{\tau}\right)\right] \\
& \left(\Delta a_{\mu}\right)_{\mathrm{LFV}}=\frac{m_{\mu}^{2}}{16 \pi^{2} \Lambda^{2}}\left[\left(\left|a_{e \mu}^{\ell}\right|^{2}+\left|v_{e \mu}^{\ell}\right|^{2}\right) h_{3}\left(x_{\mu}\right)+\frac{m_{\tau}}{m_{\mu}}\left(\left|v_{\mu \tau}^{\ell}\right|^{2}-\left|a_{\mu \tau}^{\ell}\right|^{2}\right) g_{3}\left(x_{\tau}\right)\right] \\
& \left(\Delta a_{\tau}\right)_{\mathrm{LFV}}=\frac{m_{\tau}^{2}}{16 \pi^{2} \Lambda^{2}}\left[\left(\left|a_{e \tau}^{\ell}\right|^{2}+\left|v_{e \tau}^{\ell}\right|^{2}\right) h_{3}\left(x_{\tau}\right)+\left(\left|a_{\mu \tau}^{\ell}\right|^{2}+\left|v_{\mu \tau}^{\ell}\right|^{2}\right) h_{3}\left(x_{\tau}\right)\right]
\end{aligned}
$$


where $x_{i}=m_{a}^{2} / m_{\ell_{i}}^{2}$, as before, and the loop functions $h_{i}(x)$ and $g_{i}(x)$ are reported in appendix A. Note, in particular, that $\left(\Delta a_{\ell_{i}}\right)_{\mathrm{LFV}}$ receives a chiral enhancement of order $m_{\tau} / m_{\ell_{i}}$ for $\ell_{i}=e, \mu$, hereby violating the "naive scaling". We find that the leading UVsensitive term in eq. (3.41) agrees with [50], while the sub-leading finite terms proportional to $h_{1,2}\left(x_{i}\right)$ agree with refs. [87] and [49], respectively. Moreover, the LFV contributions depicted in eq. (3.42) agree with the recent results from ref. [49].

The inspection of eqs. (3.40) and (3.42) leads to the following remarks:

- The functions $h_{1}(x), h_{2}(x)$ and $g_{3}(x)$ are identically positive, while the sign of $h_{3}(x)$ depends on the value of the ALP mass [49]. Therefore, LFC contributions from Yukawa interactions (cf. right panel of figure 1) cannot account for the $(g-2)_{\mu}$ anomaly due to the wrong sign, as noted before in refs. [49, 50, 55, 87].

- Flavor conserving contributions of Barr-Zee type (cf. left panel of figure 1) can accommodate both $(g-2)_{e}$ and $(g-2)_{\mu}$ anomalies provided $\left|c_{\gamma \gamma}\right| \gtrsim 10 \times\left|a_{\mu \mu}^{\ell}\right|$ and $\left|a_{e e}^{\ell}\right| \gtrsim 10 \times\left|a_{\mu \mu}^{\ell}\right|$, with $c_{\gamma \gamma} a_{\mu \mu}^{\ell}<0$ and $a_{e e}^{\ell} a_{\mu \mu}^{\ell}<0$. This is illustrated in figure 7 for two benchmark values for $m_{a}$, namely $m_{a}=1 \mathrm{GeV}$ and $10 \mathrm{GeV}$, and for fixed values of $a_{e e}^{\ell} / \Lambda=10 \mathrm{TeV}^{-1}$ (left panel) and $50 \mathrm{TeV}^{-1}$ (right panel). Although phenomenologically viable, this scenario requires large couplings to electrons and photons, which might be challenging to obtain in a ultraviolet complete scenario. In this plot, the different shape of the $(g-2)_{e, \mu}$ constraints can be traced back to the interplay of Barr-Zee and pure Yukawa effects, cf. eq. (3.40). For a sufficiently large (small) $a_{\mu \mu}^{\ell}$ $\left(c_{\gamma \gamma}\right)$ coupling, the Yukawa contribution - which has the wrong sign to explain the $(g-2)_{\mu}$ anomaly — tends to dominate over the Barr-Zee one, setting a lower bound on $c_{\gamma \gamma}$. This lower bound is relaxed for increasing values of $m_{a}$ as the Yukawa effects decouple faster than the Barr-Zee ones.

- LFV contributions can be especially relevant for $(g-2)_{e}$ given the large $m_{\tau} / m_{e}$ chiral enhancement in eq. (3.42). Therefore, a natural explanation of these anomalies can be obtained invoking flavor conserving contributions of Barr-Zee type for $(g-2)_{\mu}$ and LFV effects for $(g-2)_{e}$. This solution is viable as long as $\left|a_{\mu \tau}^{\ell} / a_{e \tau}^{\ell}\right| \lesssim 10^{-4}$ and $\left|a_{\tau \tau}^{\ell} / a_{e \tau}^{\ell}\right| \lesssim 10^{2}$, in order to avoid the experimental bounds from $\mu \rightarrow e \gamma$ and $\tau \rightarrow e \gamma$, respectively. ${ }^{7}$ This is illustrated in figure 8 for $m_{a}=1 \mathrm{GeV}$ (left panel) and $m_{a}=10 \mathrm{GeV}$ (right panel), for a fixed values $c_{\gamma \gamma} / \Lambda=1 \mathrm{TeV}^{-1}$. In this scenario, the $(g-2)_{\mu}$ anomaly is explained by flavor-conserving couplings (the dominant effects stemming from Barr-Zee diagrams), while $(g-2)_{e}$ is explained via chirally-enhanced LFV contributions induced by $a_{\tau e}^{\ell}$. The most important feature in these plots is the different impact of LFV processes depending on the ALP mass. In particular, for $m_{a}>m_{\tau}-m_{\mu}$, the most stringent bounds arise from $\tau \rightarrow e \gamma$, which leaves the possibility of explaining the $(g-2)$ anomalies (right panel). By contrast, for

\footnotetext{
${ }^{7}$ In principle, LFV contributions can accommodate both $(g-2)_{e}$ and $(g-2)_{\mu}$ anomalies through a unique $a_{e \mu}^{\ell}$ coupling [49]. However, we stress that such a solution would require a huge hierarchy $\left|a_{\mu \mu}^{\ell} / a_{e \mu}^{\ell}\right| \lesssim 10^{-7}$ in order to avoid the experimental bound on $\mu \rightarrow e \gamma$, making this possibility less compelling.
} 

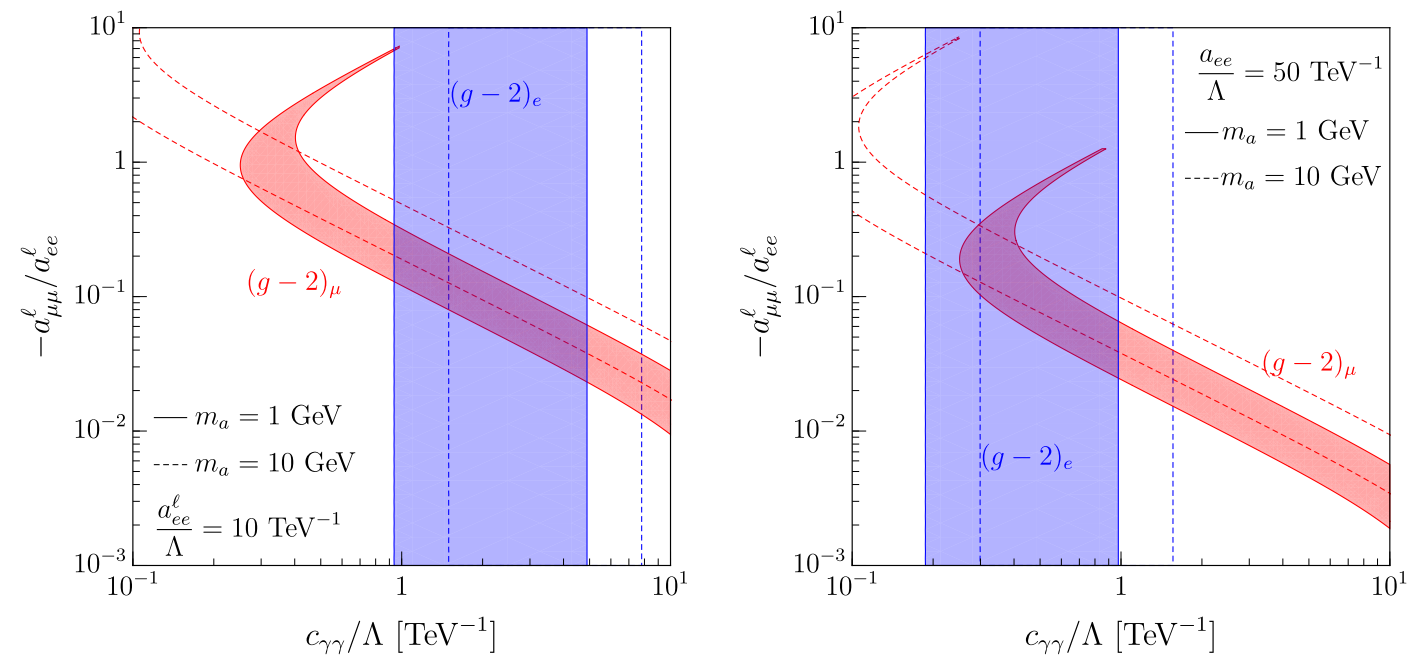

Figure 7. Allowed regions in the $-a_{\mu \mu} / a_{e e}$ vs. $c_{\gamma \gamma} / \Lambda$ plane where the $(g-2)_{\mu}$ and $(g-2)_{e}$ anomalies are accounted for at the $90 \%$ C.L. . The plots have been obtained setting $\Lambda=1 \mathrm{TeV}$, $a_{e e} / \Lambda=10 \mathrm{TeV}^{-1}$ (left) and $a_{e e} / \Lambda=50 \mathrm{TeV}^{-1}$ (right). In each plot we consider two benchmarks for the ALP mass, namely $m_{a}=1 \mathrm{GeV}$ (solid lines) and $m_{a}=10 \mathrm{GeV}$ (dashed lines).
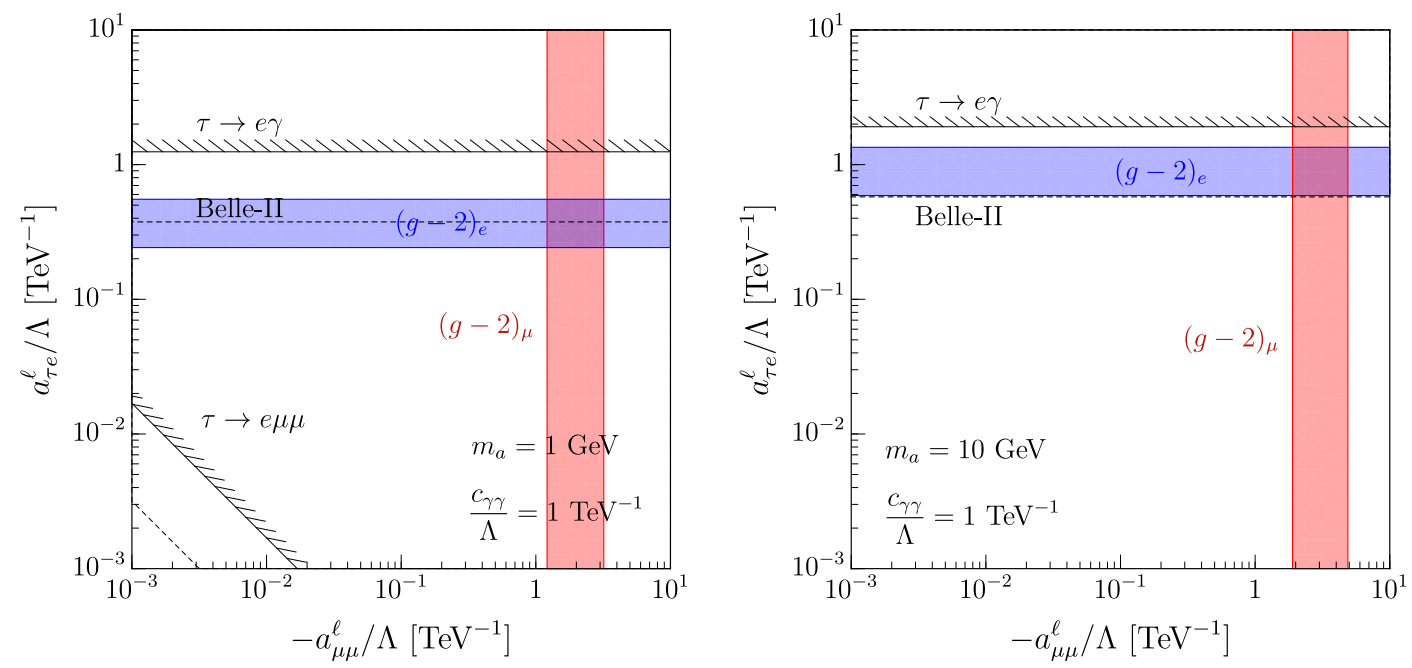

Figure 8. Allowed regions in the $a_{\tau e} / \Lambda$ vs. $-a_{\mu \mu} / \Lambda$ plane where the $(g-2)_{\mu}$ and $(g-2)_{e}$ anomalies are accounted for at the $90 \%$ C.L. . The plots have been obtained setting $\Lambda=1 \mathrm{TeV}$, $c_{\gamma \gamma} / \Lambda=1 \mathrm{TeV}^{-1}$ and $m_{a}=1 \mathrm{GeV}$ (lower left) and $m_{a}=10 \mathrm{GeV}$ (lower right). For simplicity, the other ALP couplings have been set to zero. Regions excluded by present (future) limits on $\mathcal{B}(\tau \rightarrow e \gamma)$ and $\mathcal{B}(\tau \rightarrow e \mu \mu)$ are shown by the solid (dashed) black lines, cf. table 1 .

$2 m_{\mu}<m_{a} \lesssim m_{\tau}-m_{\mu}$ (left plot) tree-level effects to $\mathcal{B}(\tau \rightarrow e \mu \mu)$ are so large that the existing limits precludes the possibility of explaining these anomalies.

- For completeness, we also comment on $(g-2)_{\tau}$. In this case, there is no parametric enhancement for LFV effects, since $m_{\tau}$ is the largest fermionic mass, therefore the dominant effects stem from LFC couplings. For $a_{\tau \tau}, v_{\tau \tau} \sim \mathcal{O}(1)$, we expect that $\left|\Delta a_{\tau}\right| \lesssim 10^{-5}$, still far from the poor sensitivity of current experiments [88, 89]. 

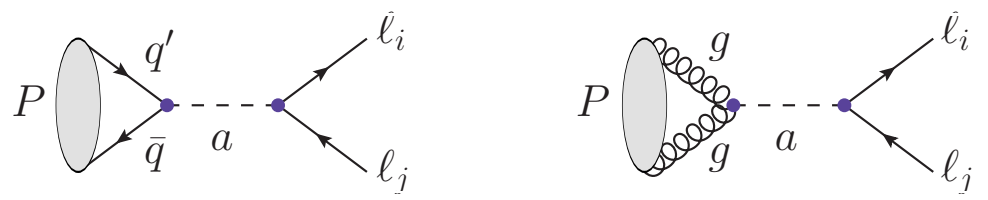

Figure 9. Diagrams contributing to the processes $P \rightarrow \ell_{i} \ell_{j}$ and $\ell_{j} \rightarrow \ell_{i} P$ via the ALP couplings to quarks (left panel) and gluons (right panel), where $P$ denotes a generic pseudoscalar meson. The latter diagram only contributes to processes involving light unflavored mesons.

\section{Hadronic processes}

LFV decays of mesons also provide a powerful probe of ALP interactions. On the one hand, they are highly complementary to the purely leptonic processes discussed in section 3 as they are sensitive to different combinations of ALP couplings. On the other hand, we expect significant experimental improvements in the coming years thanks to the effort of the NA62 [41], LHCb [42, 90] and Belle-II [43, 91] collaborations.

There are three types of hadronic processes that can be studied experimentally: (i) two body-decays $P \rightarrow \ell_{i} \ell_{j}$, (ii) semileptonic decays $P \rightarrow P^{\prime} \ell_{i} \ell_{j}$ and (iii) $P \rightarrow V \ell_{i} \ell_{j}$, where $P^{(\prime)}$ and $V$ denote generic pseudoscalar and vector mesons, respectively. Concerning the two body-decays, we focus on pseudoscalar mesons instead of vector ones, since parity conservation implies that $\left\langle 0\left|G_{\mu \nu} \widetilde{G}^{\mu \nu}\right| V\right\rangle=0$, while the relation $\left\langle 0\left|\bar{q}^{(\prime)} \gamma_{5} q\right| V\right\rangle=0$ can be derived from the Ward identities. We will derive now the most general expressions for the branching ratios of these processes and discuss their potential to probe ALP couplings. Our notation is such that leptonic charged conjugated final states are added $\ell_{i} \ell_{j} \equiv \ell_{i}^{ \pm} \ell_{j}^{\mp}=$ $\ell_{i}^{-} \ell_{j}^{+}+\ell_{i}^{+} \ell_{j}^{-}$and therefore $\mathcal{B}\left(P \rightarrow(M) \ell_{i} \ell_{j}\right)=2 \mathcal{B}\left(P \rightarrow(M) \ell_{i}^{-} \ell_{j}^{+}\right)$where $M=P^{\prime}, V$.

\section{1 $P \rightarrow \ell_{i} \ell_{j}$ and $\ell_{j} \rightarrow \ell_{i} P$}

\subsubsection{General expressions}

We start by considering the simplest LFV hadronic processes, namely the decays $P \rightarrow \ell_{i} \ell_{j}$. These processes can be induced via ALP couplings to quarks and/or gluons, as illustrated in figure 9. While the quark contribution is always present, the gluonic one can only contribute to decays of unflavored mesons, such as $P=\pi, \eta, \eta^{\prime}$.

Assuming that $m_{\ell_{j}} \gg m_{\ell_{i}}$, the branching fraction for $P \rightarrow \ell_{i} \ell_{j}$ is given by

$$
\mathcal{B}\left(P \rightarrow \ell_{i} \ell_{j}\right)=\tau_{P} \frac{m_{P} m_{\ell_{j}}^{2}}{4 \pi \Lambda^{4}} \frac{\left|\mathcal{N}_{P}\right|^{2}}{\left(m_{P}^{2}-m_{a}^{2}\right)^{2}+m_{a}^{2} \Gamma_{a}^{2}}\left(1-\frac{m_{\ell_{j}}^{2}}{m_{P}^{2}}\right)^{2}\left|s_{i j}^{\ell}\right|^{2},
$$

where $\mathcal{N}_{P} \equiv \Lambda\left\langle 0\left|\mathcal{L}_{\text {eff }}^{D \leq 5}\right| P\right\rangle$ is a function of the ALP couplings to quarks and gluons, and of the relevant hadronic parameters, which can be expressed as follows

$$
\mathcal{N}_{\mathcal{P}}=16 \pi^{2} c_{g g} a_{P}-i a_{i j}^{q}\left(m_{q_{i}}+m_{q_{j}}\right)\left\langle 0\left|\bar{q}_{i} \gamma_{5} q_{j}\right| P\right\rangle,
$$

where the summation over quark-flavor indices is implicit and the hadronic constant $a_{P}$ is defined as

$$
a_{P} \equiv\left\langle 0\left|\frac{\alpha_{s}}{4 \pi} G_{\mu \nu} \widetilde{G}^{\mu \nu}\right| P\right\rangle
$$


In eq. (4.2), the matrix element $\left\langle 0\left|\bar{q}_{i} \gamma_{5} q_{j}\right| P\right\rangle$ depends on the relevant meson decay constants which should be determined together with $a_{P}$ by non-perturbative means, as will be discussed below.

If kinematically allowed, the same couplings generating $P \rightarrow \ell_{i} \ell_{j}$ also generate the inverse process $\ell_{j} \rightarrow P \ell_{i}$. The corresponding branching fraction is

$$
\mathcal{B}\left(\ell_{j} \rightarrow P \ell_{i}\right)=\tau_{\ell_{i}} \frac{m_{\ell_{j}}^{3}}{16 \pi \Lambda^{4}} \frac{\left|\mathcal{N}_{P}\right|^{2}}{\left(m_{P}^{2}-m_{a}^{2}\right)^{2}+m_{a}^{2} \Gamma_{a}^{2}}\left(1-\frac{m_{P}^{2}}{m_{\ell_{j}}^{2}}\right)^{2}\left|s_{i j}^{\ell}\right|^{2},
$$

which depends on the parameter $\mathcal{N}_{P}$ defined above.

\subsubsection{Hadronic inputs: $\mathcal{N}_{P}$}

The next step is to derive the expression for $\mathcal{N}_{P}$, defined in eq. (4.2), for each pseudoscalar meson. This quantity depends on the pseudoscalar density, $\left\langle 0\left|q_{i} \gamma_{5} q_{j}\right| P\right\rangle$, as well as on the anomaly matrix element, parameterized by $a_{P}$ in eq. (4.3).

Flavored mesons. We start by considering heavy flavored mesons such a $D^{0}$ and $B_{(s)}$. In this case, the only non-vanishing contribution comes from the second term in eq. (4.2). By expressing the heavy-light meson as $P=\bar{Q} q$, the axial matrix element reads

$$
\left\langle 0\left|\bar{Q} \gamma^{\mu} \gamma_{5} q\right| P(p)\right\rangle=i f_{P} p^{\mu}
$$

from which one can show that

$$
\left\langle 0\left|\bar{Q} \gamma_{5} q\right| P\right\rangle=-i \frac{m_{P}^{2} f_{P}}{m_{q}+m_{Q}}
$$

where $f_{P}$ is the $P$-meson decay constant. By replacing this expression in eq. (4.2), we obtain

$$
\begin{aligned}
\mathcal{N}_{D} & =-a_{12}^{u} f_{D} m_{D}^{2}, \\
\mathcal{N}_{B_{d}} & =-a_{13}^{d} f_{B} m_{B_{d}}^{2}, \\
\mathcal{N}_{B_{s}} & =-a_{23}^{d} f_{B_{s}} m_{B_{s}}^{2} .
\end{aligned}
$$

In these expressions, the only needed inputs are $f_{P}$, which have been determined in all cases by means of numerical simulations of QCD on the lattice, cf. table 2. Similarly, in the kaon system, we define $\left|K_{L(S)}\right\rangle=\left(\left|K^{0}\right\rangle \pm\left|\overline{K^{0}}\right\rangle\right) / \sqrt{2}$ and write

$$
\begin{aligned}
& \mathcal{N}_{K_{L}}=-\sqrt{2} \operatorname{Re}\left[a_{12}^{d}\right] m_{K^{0}}^{2} f_{K}, \\
& \mathcal{N}_{K_{S}}=-i \sqrt{2} \operatorname{Im}\left[a_{12}^{d}\right] m_{K^{0}}^{2} f_{K}
\end{aligned}
$$

showing that $K_{L(S)}$ leptonic decays can probe either the real or imaginary part of $a_{12}^{d}$. 


\begin{tabular}{|c|cc|}
\hline Quantity & Value $[\mathrm{MeV}]$ & Ref. \\
\hline$f_{\pi}$ & $130.2(0.8)$ & {$[92]$} \\
$f_{K}$ & $155.7(0.3)$ & {$[92]$} \\
$f_{D}$ & $212.0(0.7)$ & {$[92]$} \\
$f_{B_{d}}$ & $190.0(1.3)$ & {$[92]$} \\
$f_{B_{s}}$ & $230.3(1.3)$ & {$[92]$} \\
\hline$f_{\eta_{c}}$ & $391(4)$ & {$[93]$} \\
$f_{\eta_{b}}$ & $667(6)$ & {$[94]$} \\
\hline
\end{tabular}

Table 2. Decay constants computed by means of numerical simulations of QCD on the lattice.

Pseudoscalar quarkonia states. For the heavy quarkonia states $\eta_{c}$ and $\eta_{b}$, we find in a similar way that

$$
\begin{aligned}
& \mathcal{N}_{\eta_{c}}=16 \pi^{2} c_{g g} a_{\eta_{c}}-a_{22}^{u} f_{\eta_{c}} m_{\eta_{c}}^{2}, \\
& \mathcal{N}_{\eta_{b}}=16 \pi^{2} c_{g g} a_{\eta_{b}}-a_{33}^{d} f_{\eta_{b}} m_{\eta_{b}}^{2}
\end{aligned}
$$

where $f_{\eta_{c}}$ and $f_{\eta_{b}}$ are also listed in table 2. The anomaly contribution is also present in this case, but it is sub-dominant since these particles are much heavier than $\Lambda_{\mathrm{QCD}}$.

Light unflavored mesons. Finally, we discuss the more subtle case of $\pi^{0}$ and $\eta^{(\prime)}$ mesons. For pions, one can use the exact isospin limit to derive the pseudoscalar density, while the anomaly contribution can be obtained by taking the divergence of the axial current $[95,96]$. We find that

$$
\mathcal{N}_{\pi}=-\frac{f_{\pi} m_{\pi}^{2}}{\sqrt{2}}\left(a_{11}^{u}-a_{11}^{d}\right)-16 \pi^{2} \frac{1-z}{1+z} \frac{f_{\pi} m_{\pi}^{2}}{\sqrt{2}} c_{g g}
$$

where $z=m_{u} / m_{d}$, in such a way that the anomaly contribution vanishes in the isospin conserving limit, $m_{u}=m_{d}$. For $\eta^{(\prime)}$, the anomaly contribution plays an even more important role. By denoting $q=u, d$ and taking $m_{q}=\left(m_{u}+m_{d}\right) / 2$, the pseudoscalar densities can be parameterized as

$$
\begin{aligned}
2 m_{q}\left\langle 0\left|\bar{q} \gamma_{5} q\right| \eta^{(\prime)}\right\rangle & =-\frac{i}{\sqrt{2}} h_{\eta^{(\prime)}}^{q}, \\
2 m_{s}\left\langle 0\left|\bar{s} \gamma_{5} s\right| \eta^{(\prime)}\right\rangle & =-i h_{\eta^{(\prime)}}^{s},
\end{aligned}
$$

where $h_{\eta^{(\prime)}}^{q}$ and $h_{\eta^{(\prime)}}^{s}$ are decay constants. ${ }^{8}$ These definitions allow us to write

$$
\mathcal{N}_{\eta^{(\prime)}}=16 \pi^{2} c_{g g} a_{\eta^{(\prime)}}-\left(a_{11}^{u}+a_{11}^{d}\right) \frac{h_{\eta^{(\prime)}}^{q}}{\sqrt{2}}-a_{22}^{d} h_{\eta^{(\prime)}}^{s} .
$$

\footnotetext{
${ }^{8}$ Note that the axial and pseudoscalar densities are not directly related to the same decay constant for $\eta$ and $\eta^{\prime}$ since the anomalous contribution is relevant in this case.
} 


\begin{tabular}{|c|ccc|}
\hline$P$ & $h_{P}^{q}\left[\mathrm{GeV}^{3}\right]$ & $h_{P}^{s}\left[\mathrm{GeV}^{3}\right]$ & $a_{P}\left[\mathrm{GeV}^{3}\right]$ \\
\hline$\eta$ & $0.001(3)$ & $-0.055(3)$ & $-0.022(2)$ \\
$\eta^{\prime}$ & $0.001(2)$ & $0.068(5)$ & $-0.057(2)$ \\
\hline
\end{tabular}

Table 3. Hadronic inputs for $\eta$ and $\eta^{\prime}$ obtained in refs. $[97,98]$ by using the FKS mixing scheme.

The best available computation of $a_{\eta^{(\prime)}}, h_{\eta^{(\prime)}}^{q}$ and $h_{\eta^{(\prime)}}^{s}$ relies on the so-called FeldmannKroll-Stech (FKS) mixing scheme [97]. This phenomenological approach is based on the assumption that the states $\left.\left|\eta_{q}\right\rangle=(|u \bar{u}+| d \bar{d}\rangle\right) / \sqrt{2}$ and $\left|\eta_{s}\right\rangle=|s \bar{s}\rangle$ only mix through the anomaly. In this case, by using inputs such as the mixing angle between $\eta$ and $\eta^{\prime}$, the authors of refs. [97, 98] obtained the inputs collected in table 3.

\section{2 $P \rightarrow P^{\prime} \ell_{i} \ell_{j}$}

\subsubsection{General expressions}

The next processes we consider are semileptonic decays of the type $P \rightarrow P^{\prime} \ell_{i} \ell_{j}$. These processes can be induced via the ALP couplings to quarks, cf. figure 9 with $q \neq q^{\prime}$, and are complementary to the processes $P \rightarrow \ell_{i} \ell_{j}$ described above due to the parity symmetry. More specifically, since these decays arise from the underlying transition $q_{k} \rightarrow q_{l} \ell_{i}^{-} \ell_{j}^{+}$(with $i \neq j$ and $k \neq l$ ), the relevant hadronic matrix elements read

$$
\left\langle P^{\prime}(k)\left|\bar{q}_{l} \gamma_{5} q_{k}\right| P(p)\right\rangle=0, \quad\left\langle P^{\prime}(k)\left|\bar{q}_{l} q_{k}\right| P(p)\right\rangle=f_{0}\left(q^{2}\right) \frac{m_{P}^{2}-m_{P^{\prime}}^{2}}{m_{q_{k}}-m_{q_{l}}}
$$

where $q^{2}=(p-k)^{2}$ and $f_{0}\left(q^{2}\right) \equiv f_{0}^{P \rightarrow P^{\prime}}\left(q^{2}\right)$ denotes the $P \rightarrow P^{\prime}$ scalar form factor. Since the pseudoscalar matrix element vanishes, these decays can only constrain the vector ALP couplings $v_{k l}^{q}$. The general branching fraction is then given by [99]

$$
\frac{\mathrm{d} \mathcal{B}}{\mathrm{d} q^{2}}\left(P \rightarrow P^{\prime} \ell_{j} \ell_{i}\right)=\frac{\tau_{P} m_{P} m_{\ell_{j}}^{2}}{64 \pi^{3}} \frac{q^{2} \lambda_{P^{\prime}}^{1 / 2} f_{0}\left(q^{2}\right)^{2}}{\left(q^{2}-m_{a}^{2}\right)^{2}+m_{a}^{2} \Gamma_{a}^{2}}\left(1-\frac{m_{\ell_{j}}^{2}}{q^{2}}\right)^{2}\left(1-\frac{m_{P^{\prime}}^{2}}{m_{P}^{2}}\right)^{2} \frac{\left|v_{k l}^{q}\right|^{2}\left|s_{i j}^{\ell}\right|^{2}}{\Lambda^{4}},
$$

where we have assumed once again $m_{\ell_{j}} \gg m_{\ell_{i}}$, and the phase-space function is given by $\lambda_{P^{\prime}} \equiv \lambda\left(m_{P}, m_{P^{\prime}}, \sqrt{q^{2}}\right)$. This expression can be directly applied to the decays $D \rightarrow \pi$, $D_{s} \rightarrow K$ and $B \rightarrow K$, among others. The only subtle case regards kaon decays, for which the above expression should be amended by replacing $\left|v_{k l}^{q}\right|^{2}$ by

$$
\begin{array}{ll}
K^{+} \rightarrow \pi^{+}: & \left|v_{21}^{d}\right|^{2}, \\
K_{L} \rightarrow \pi^{0}: & \operatorname{Re}\left[v_{21}^{d}\right]^{2}, \\
K_{S} \rightarrow \pi^{0}: & \operatorname{Im}\left[v_{21}^{d}\right]^{2} .
\end{array}
$$

In other words, the different neutral kaon decays can probe either the real or imaginary parts of the Wilson coefficients, depending on their $C P$ properties.

The largest contribution to $\mathcal{B}\left(P \rightarrow P^{\prime} \ell_{i} \ell_{j}\right)$ arises when the ALP can be produced on-shell, i.e. for $m_{a} \in\left(m_{\ell_{i}}+m_{\ell_{j}}, m_{P}-m_{P^{\prime}}\right)$. In this case, the above expression can be 
simplified by means of the narrow-width approximation

$$
\mathcal{B}\left(P \rightarrow P^{\prime} a \rightarrow P^{\prime} \ell_{i} \ell_{j}\right)=\mathcal{B}\left(P \rightarrow P^{\prime} a\right) \mathcal{B}\left(a \rightarrow \ell_{i} \ell_{j}\right)
$$

where

$$
\mathcal{B}\left(P \rightarrow P^{\prime} a\right)=\frac{\tau_{P} m_{P} f_{0}\left(m_{a}^{2}\right)^{2}}{16 \pi}\left(1-\frac{m_{P^{\prime}}^{2}}{m_{P}^{2}}\right)^{2} \frac{\left|v_{k l}^{q}\right|^{2}}{\Lambda^{2}} \lambda^{1 / 2}\left(m_{P}, m_{P}^{\prime}, m_{a}\right),
$$

and the leptonic branching fraction is given in eq. (3.23). ${ }^{9}$

\subsubsection{Hadronic inputs}

The last theoretical input needed in the above expression is the form factor $f_{0}$. The latest LQCD results are summarized in ref. [100]. For the $B \rightarrow \pi$ and $B \rightarrow K$ transitions, for which LQCD form factors are not available in the full $q^{2}$-range, we use results from light-cone sum rules [101].

\section{$4.3 P \rightarrow V \ell_{i} \ell_{j}$}

\subsubsection{General expressions}

We now turn to $P \rightarrow V$ semileptonic decays, with $V$ being a generic vector meson, which turn out to be complementary to the observables described above. In this case, the relevant matrix elements are

$$
\left\langle V(k)\left|\bar{q}_{l} \gamma_{5} q_{k}\right| P(p)\right\rangle=-i(\varepsilon \cdot q) \frac{2 m_{V}}{m_{b}+m_{s}} A_{0}\left(q^{2}\right), \quad\left\langle V(k)\left|\bar{q}_{l} q_{k}\right| P(p)\right\rangle=0,
$$

where $q^{2}=(p-k)^{2}, \varepsilon^{\mu}$ denotes the $V$-meson polarization, and $A_{0} \equiv A_{0}^{P \rightarrow V}$ stands for the pseudoscalar $P \rightarrow V$ form factor. Since the scalar matrix element vanishes in this case, these decays can only probe the axial coupling $a_{k l}^{q}$, differently from the $P \rightarrow P^{\prime}$ processes described above, that are only sensitive to $v_{k l}^{q}$. The general expression for their branching fraction can be recast from ref. [99], giving

$$
\frac{\mathrm{d} \mathcal{B}}{\mathrm{d} q^{2}}\left(P \rightarrow V \ell_{i} \ell_{j}\right)=\frac{\tau_{P} m_{\ell_{j}}^{2}}{64 \pi^{3} m_{P}^{3}} \frac{\left|a_{k l}^{q}\right|^{2}\left|s_{i j}^{\ell}\right|^{2}}{\Lambda^{4}}\left(1-\frac{m_{\ell_{j}}^{2}}{q^{2}}\right)^{2} \frac{q^{2} \lambda_{V}^{3 / 2}\left(q^{2}\right) A_{0}\left(q^{2}\right)^{2}}{\left(q^{2}-m_{a}^{2}\right)^{2}+m_{a}^{2} \Gamma_{a}^{2}},
$$

where $\lambda_{V}\left(q^{2}\right)=\lambda\left(m_{P}, m_{V}, \sqrt{q^{2}}\right)$.

For on-shell ALP production, i.e. $m_{a} \in\left(m_{\ell_{i}}+m_{\ell_{j}}, m_{P}-m_{V}\right)$, the above formula can be simplified as

$$
\mathcal{B}\left(P \rightarrow V a \rightarrow V \ell_{i} \ell_{j}\right)=\mathcal{B}(P \rightarrow V a) \mathcal{B}\left(a \rightarrow \ell_{i} \ell_{j}\right),
$$

where the ALP branching fraction is given in eq. (3.23) and

$$
\mathcal{B}(P \rightarrow V a)=\frac{\tau_{P} A_{0}\left(m_{a}^{2}\right)^{2}}{16 \pi m_{P}^{3}} \frac{\left|a_{k l}^{q}\right|^{2}}{\Lambda^{2}} \lambda_{V}^{3 / 2}\left(m_{a}^{2}\right) .
$$

\footnotetext{
${ }^{9}$ Note that our notation is such that $\mathcal{B}\left(a \rightarrow \ell_{i} \ell_{j}\right) \equiv \mathcal{B}\left(a \rightarrow \ell_{i}^{ \pm} \ell_{j}^{\mp}\right)=2 \mathcal{B}\left(a \rightarrow \ell_{i}^{-} \ell_{j}^{+}\right)$.
} 


\subsubsection{Hadronic inputs}

The most relevant $P \rightarrow V$ transition for our study is $B \rightarrow K^{*}$. Since the nedeed form factor is not yet available from LQCD simulations in the full $q^{2}$ range, we consider the combination of LQCD and light-cone sum rules results from ref. [102].

\subsection{Numerical results and discussion}

In this section we derive the limits from existing data and compare the sensitivity of the different decay channels listed above. The present experimental constraints are collected in table 4 along with the future prospects when available.

Let us first consider the ALP couplings to gluons, $c_{a g g}$, which can trigger LFV processes involving light unflavored mesons, namely $\pi^{0}, \eta$ and $\eta^{\prime}$. Starting with the $\mu \rightarrow e$ transition, the only kinematically-allowed processes are $\pi^{0} \rightarrow \mu e$ and $\eta^{(\prime)} \rightarrow \mu e$. By combining eq. (4.1) with the hadronic inputs in eq. (4.14) and (4.17), and neglecting the ALP couplings to quarks, we find that

$$
\mathcal{B}(P \rightarrow \mu e)=c_{P}^{\mu e}\left|s_{12}^{\ell}\right|^{2}\left(\frac{c_{g g}}{1 / 16 \pi^{2}}\right)^{2}\left(\frac{\Lambda}{1 \mathrm{TeV}}\right)^{-4}\left(\frac{m_{P}^{2}}{m_{P}^{2}-m_{a}^{2}}\right)^{2},
$$

with

$$
\left\{c_{\pi^{0}}^{\mu e}, c_{\eta}^{\mu e}, c_{\eta^{\prime}}^{\mu e}\right\} \simeq\{2.6,0.02,0.2\} \times 10^{-13} .
$$

From these expressions, given the current experimental sensitivity depicted in table 4 , as well as the existing limits on $s_{12}^{\ell}$ derived from leptonic observables in section 3 , we conclude that such processes are not promising probes of ALPs. The only exception are the very narrow regions around $m_{a} \approx m_{P}$, where a resonant contribution is produced. Similar conclusions can be obtained by considering, instead of $c_{g g}$, the axial ALP couplings to quarks with appropriate flavor indices.

The situation is much more promising for the $\tau \rightarrow e$ and $\tau \rightarrow \mu$ transitions. In this case, the available processes are $\tau \rightarrow l \pi^{0}$ and $\tau \rightarrow l \eta^{(\prime)}$, with $l=e, \mu$. By only keeping the contributions driven by $c_{a g g}$ and by focusing on the decays into muons, we find that

$$
\mathcal{B}(\tau \rightarrow \mu P) \approx c_{P}^{\mu \tau}\left|s_{23}^{\ell}\right|^{2}\left(\frac{c_{g g}}{1 / 16 \pi^{2}}\right)^{2}\left(\frac{\Lambda}{1 \mathrm{TeV}}\right)^{-4}\left(\frac{m_{\pi^{0}}^{2}}{m_{\pi^{0}}^{2}-m_{a}^{2}}\right)^{2},
$$

with

$$
\left\{c_{\pi^{0}}^{\mu \tau}, c_{\eta}^{\mu \tau}, c_{\eta^{\prime}}^{\mu \tau}\right\} \simeq\{0.05,2.0,1.0\} \times 10^{-4},
$$

which are considerably larger than the values found in eq. (4.30). These decay modes are particularly interesting given the expected experimental resolutions at Belle-II, which are going to improve the present limits by at least one order of magnitude [43], cf. table 4. To understand why the processes $\tau \rightarrow \ell P$ are more sensitive to new physics than $P \rightarrow \mu e$, one should compare the total lifetime of the decaying particles. More precisely, one finds that

$$
\frac{\tau_{\tau}}{\tau_{\pi}} \approx 3.4 \times 10^{3}, \quad \frac{\tau_{\tau}}{\tau_{\eta}} \approx 5.8 \times 10^{5}, \quad \text { and } \quad \frac{\tau_{\tau}}{\tau_{\eta^{\prime}}} \approx 8.6 \times 10^{7}
$$




\begin{tabular}{|c|ccc|}
\hline Decay mode & Exp. limit & Future prospects & Ref. \\
\hline$\pi^{0} \rightarrow \mu^{\mp} e^{ \pm}$ & $3.6 \times 10^{-10}$ & - & {$[82]$} \\
$\eta \rightarrow \mu^{\mp} e^{ \pm}$ & $6 \times 10^{-6}$ & - & {$[82]$} \\
$\eta^{\prime} \rightarrow \mu^{\mp} e^{ \pm}$ & $4.7 \times 10^{-4}$ & - & {$[82]$} \\
$K_{L} \rightarrow \mu^{\mp} e^{ \pm}$ & $4.7 \times 10^{-12}$ & - & {$[82]$} \\
$K^{+} \rightarrow \pi^{+} \mu^{+} e^{-}$ & $1.3 \times 10^{-11}$ & $\approx 10^{-12}$ & {$[82]$} \\
$D \rightarrow \mu^{ \pm} e^{\mp}$ & $1.3 \times 10^{-8}$ & - & {$[82]$} \\
$B_{d} \rightarrow \mu^{\mp} e^{ \pm}$ & $1.0 \times 10^{-9}$ & $\approx 2 \times 10^{-10}$ & {$[82]$} \\
$B_{s} \rightarrow \mu^{\mp} e^{ \pm}$ & $5.4 \times 10^{-9}$ & $\approx 8 \times 10^{-10}$ & {$[82]$} \\
$B^{+} \rightarrow K^{+} \mu^{+} e^{-}$ & $6.4 \times 10^{-9}$ & - & {$[104]$} \\
$B^{0} \rightarrow K^{*} \mu^{\mp} e^{ \pm}$ & $1.8 \times 10^{-7}$ & - & {$[82]$} \\
\hline$\tau \rightarrow e \pi^{0}$ & $8.0 \times 10^{-8}$ & $\approx 4 \times 10^{-10}$ & {$[82]$} \\
$\tau \rightarrow e \eta$ & $9.2 \times 10^{-8}$ & $\approx 9 \times 10^{-10}$ & {$[82]$} \\
$\tau \rightarrow e \eta^{\prime}$ & $1.6 \times 10^{-7}$ & $\approx 7 \times 10^{-10}$ & {$[82]$} \\
$\tau \rightarrow e K_{S}$ & $2.6 \times 10^{-8}$ & $\approx 5 \times 10^{-10}$ & {$[82]$} \\
$B_{d} \rightarrow \tau^{ \pm} e^{\mp}$ & $2.8 \times 10^{-5}$ & $\approx 1.6 \times 10^{-5}$ & {$[82]$} \\
$B^{+} \rightarrow K^{+} \tau^{ \pm} e^{\mp}$ & $3 \times 10^{-5}$ & $\approx 2.1 \times 10^{-6}$ & {$[82]$} \\
\hline$\tau \rightarrow \mu \pi^{0}$ & $1.1 \times 10^{-7}$ & $\approx 5 \times 10^{-10}$ & {$[82]$} \\
$\tau \rightarrow \mu \eta$ & $6.5 \times 10^{-8}$ & $\approx 4 \times 10^{-10}$ & {$[82]$} \\
$\tau \rightarrow \mu \eta^{\prime}$ & $1.3 \times 10^{-7}$ & $\approx 8 \times 10^{-10}$ & {$[82]$} \\
$\tau \rightarrow \mu K_{S}$ & $2.3 \times 10^{-8}$ & $\approx 4 \times 10^{-10}$ & {$[82]$} \\
$B_{d} \rightarrow \tau^{ \pm} \mu^{\mp}$ & $4.2 \times 10^{-5}$ & $\approx 1.3 \times 10^{-5}$ & {$[105]$} \\
$B_{s} \rightarrow \tau^{ \pm} \mu^{\mp}$ & $1.4 \times 10^{-5}$ & - & {$[105]$} \\
$B^{+} \rightarrow K^{+} \tau^{ \pm} \mu^{\mp}$ & $4.8 \times 10^{-5}$ & $\approx 3.3 \times 10^{-6}$ & {$[82]$} \\
\hline & & & \\
\hline$\tau$ & & - & $\approx$ \\
\hline
\end{tabular}

Table 4. Most relevant experimental limits on LFV $\tau$ and leptonic meson decays [82] and future prospects for NA62 [41], LHCb [42, 90] and Belle-II [43, 91].

which can be understood from the fact that $\tau$ 's can only decay through weak interactions, differently than $\pi^{0}$ and $\eta^{(\prime)}$. For that reason, $\tau$ decays are much better probes of new physics than light-meson decays.

To further explore the potential of $\tau$ decays to constrain ALPs, in figure 10 we plot $\mathcal{B}(\tau \rightarrow \mu P)$, normalized to $\left|s_{23}^{\ell}\right|^{2}$, as a function of $m_{a}$ in two scenarios: (i) $c_{a g g}=1 /\left(16 \pi^{2}\right)$ and (ii) $a_{i i}^{d}=a_{i i}^{u}=1$, with the other couplings taken to be zero. From these plots, we see that the largest branching fractions are obtained at the resonances, i.e. $m_{a} \approx m_{P}$. However, large branching fractions can also be attained for other masses. Notice, also, that these decays modes have a complementary sensitivity to $c_{a g g}, a_{i i}^{d}$ and $a_{i i}^{u}$, as it can be seen by comparing the left and right panels of figure 10 . 

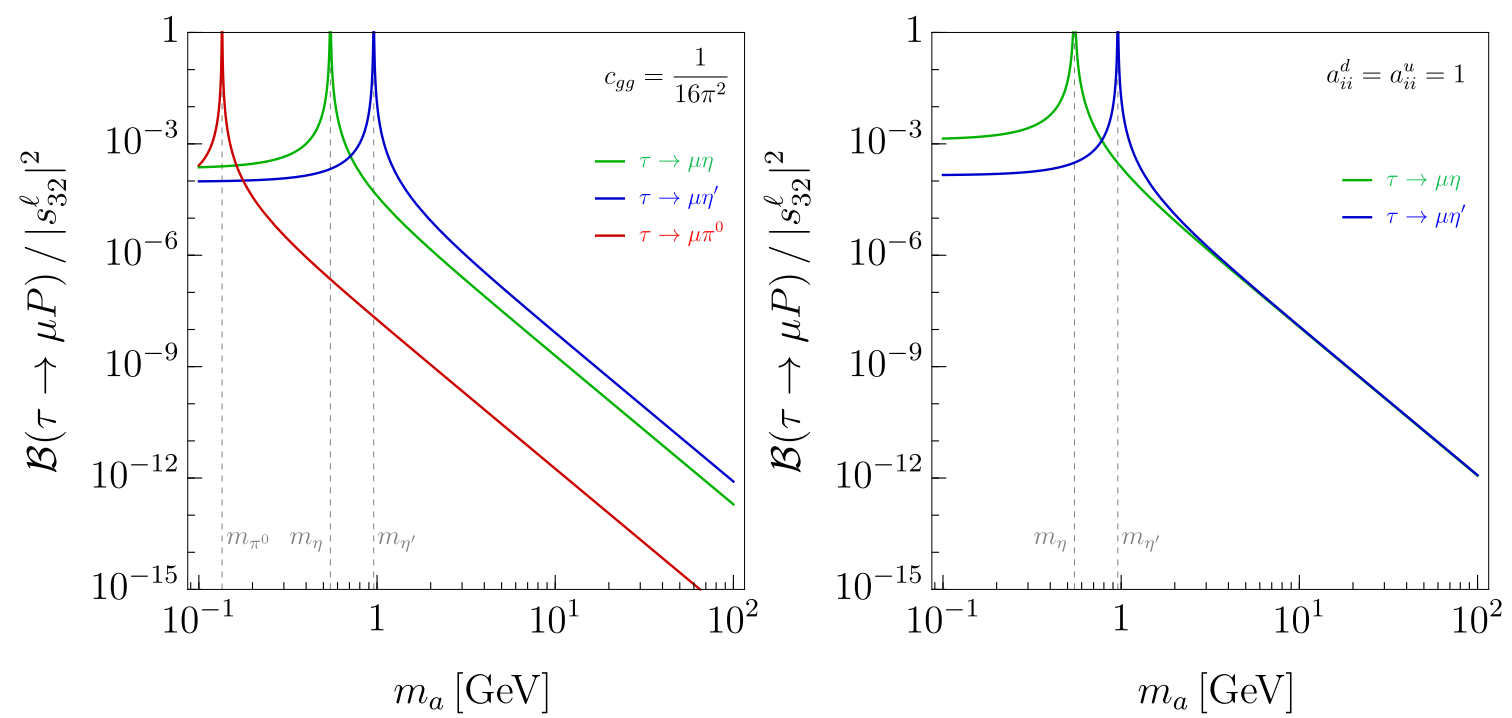

Figure 10. Normalized branching fraction $\mathcal{B}(\tau \rightarrow \mu P) /\left|s_{23}^{\ell}\right|^{2}$ plotted against $m_{a}$ for $P=\pi^{0}$ and $\eta^{(\prime)}$ in two benchmark scenarios, namely $c_{g g}=1 /\left(16 \pi^{2}\right)$ (left panel) and $a_{i i}^{d}=a_{i i}^{u}=1$ (right panel).

Next, we discuss the potential of (semi)leptonic kaon and $B$-meson decays to probe ALP couplings. These decay modes are very sensitive to NP contributions due to their quark-flavor changing nature. Furthermore, there is a rich experimental program at NA62, $\mathrm{LHCb}$ and Belle-II experiments that will improve the experimental sensitivity on many observables. Unlike the processes discussed above, (semi)leptonic decays can only probe the flavor violating ALP couplings to quarks, $a_{i j}^{q}$ and $v_{i j}^{q}$, with $i \neq j$. Leptonic decays are sensitive to axial couplings, while semileptonic decays can probe either the axial or vector ones, depending on the spin of the meson in the final state. For this reason, these processes provide complementary information on the NP couplings. To quantitatively compare the different decay modes, we assume that $a_{i j}^{d}=v_{i j}^{d}=10^{-3}$ and plot the branching fractions normalized by the LFV coupling as a function of $m_{a}$. This is shown in figure 11 and 12 for kaon and $B_{(s)}$-meson observables, respectively. We find that the semileptonic rates are always the largest ones in the resonant regions, while the leptonic ones are typically dominant for large and/or small values of $m_{a}$, outside the resonant region.

To compare the sensitivity of kaon and $B$-meson decays channels, we consider a benchmark model that allows us to connect different quark transitions. We assume that the matrices $a^{d}$ and $v^{d}$ satisfy

$$
a_{i j}^{d}=v_{i j}^{d} \simeq c_{d} V_{t i} V_{t j}^{*},
$$

where $V$ denotes the CKM matrix and $c_{d}$ is a constant. Such relation is predicted, for instance, from electroweak loops in models with predominant ALP couplings to the $W$ boson and/or the top-quark $[39,40]$. The constraints on $s_{i j}^{\ell}$ derived from kaon and $B$ meson decays are shown in figure 13 for a fixed value of $c_{d}$. To derive these constraints, one should also specify the total ALP width, which greatly affects the constraints derived in the resonant regions. In figure 13, we assume for illustration a constant value $\Gamma_{a}=10^{-6} \mathrm{GeV} .{ }^{10}$ Smaller (larger) values would imply stronger (weaker) constraints in these regions, without

\footnotetext{
${ }^{10}$ See ref. [103] for a data-driven approach proposed to determine $\Gamma(a \rightarrow$ hadrons $)$ for ALP masses in the $\mathrm{GeV}$ range.
} 


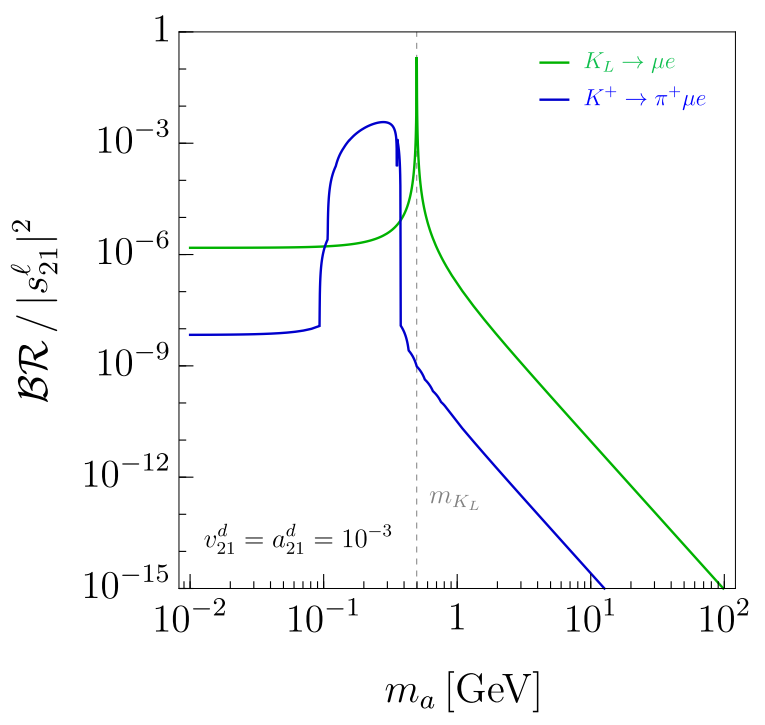

Figure 11. $\mathcal{B}\left(K^{+} \rightarrow \pi^{+} \mu e\right) /\left|s_{21}^{\ell}\right|^{2}$ (blue line) and $\mathcal{B}\left(K_{L} \rightarrow \mu e\right) /\left|s_{12}^{\ell}\right|^{2}$ (green line) are plotted against $m_{a}$. The ALP couplings to quarks are taken to be $v_{21}^{d}=a_{21}^{d}=10^{-3}$, while the ALP width is fixed to $\Gamma_{a}=10^{-6} \mathrm{GeV}$ for illustration. Different values of $\Gamma_{a}$ would imply a shift of the semileptonic rate in the resonant region.
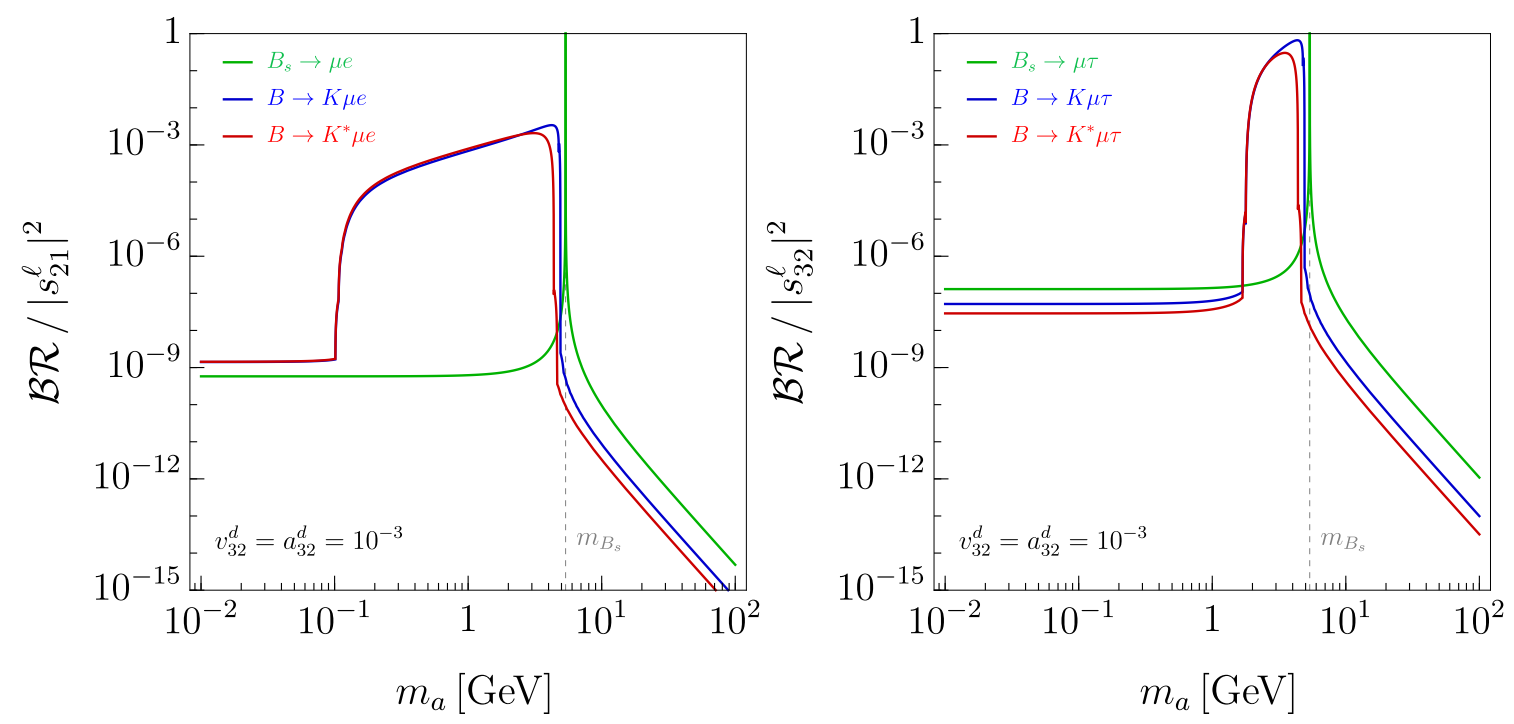

Figure 12. $\mathcal{B}\left(B \rightarrow K \ell_{i} \ell_{j}\right) /\left|s_{i j}^{\ell}\right|^{2}$ (green line), $\mathcal{B}\left(B \rightarrow K^{*} \ell_{i} \ell_{j}\right) /\left|s_{i j}^{\ell}\right|^{2}$ (blue line) and $\mathcal{B}\left(B_{s} \rightarrow\right.$ $\left.\ell_{i} \ell_{j}\right) /\left|s_{i j}^{\ell}\right|^{2}$ (red line) are plotted against $m_{a}$ for $(i, j)=(1,2)$ (left panel) and $(2,3)$ (right panel). The ALP couplings to quarks are taken to be $v_{32}^{d}=a_{32}^{d}=10^{-3}$, while the ALP width is fixed to $\Gamma_{a}=10^{-6} \mathrm{GeV}$ for illustration. 

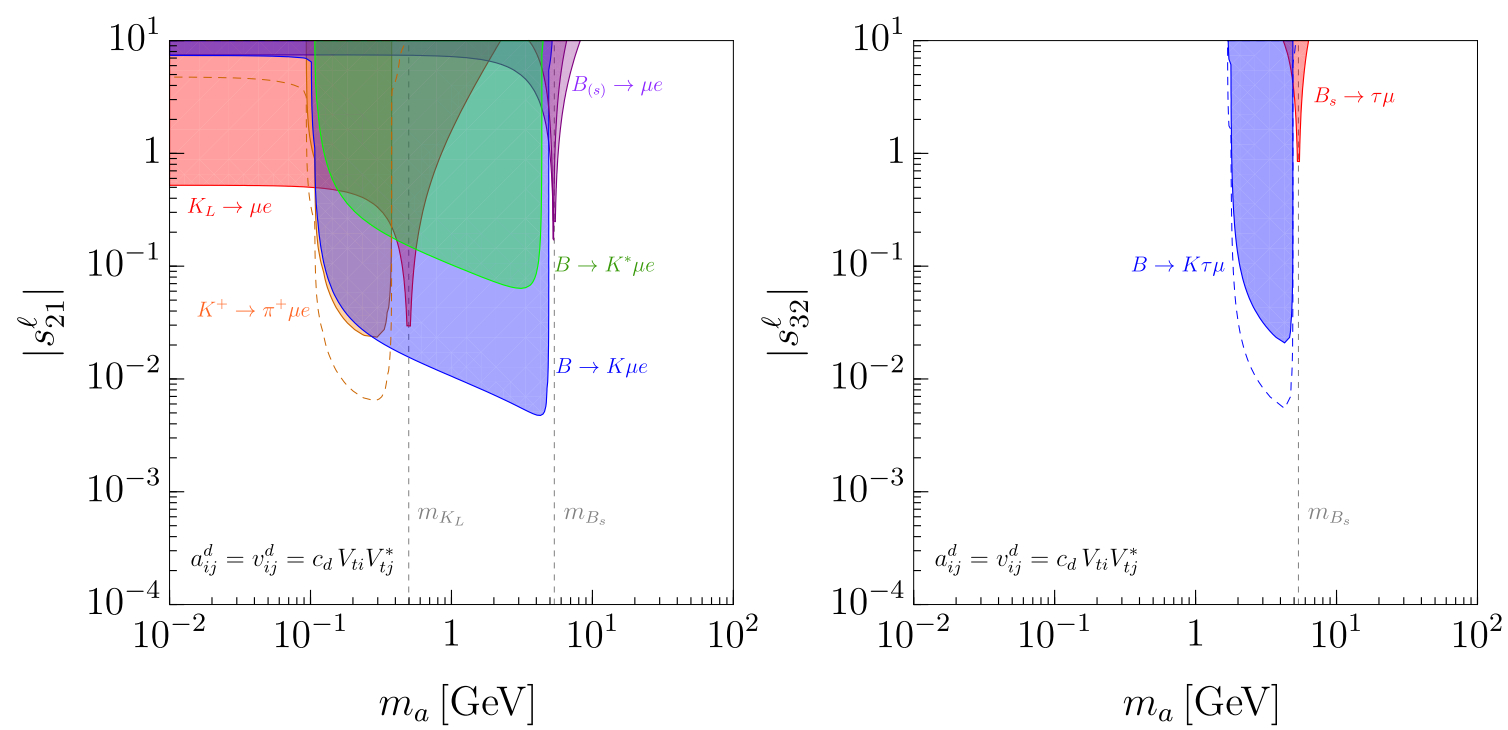

Figure 13. Constraints on $\left|s_{21}^{\ell}\right|$ (left panel) and $\left|s_{32}^{\ell}\right|$ (right panel) as a function of the ALP mass obtained from the hadronic probes listed in table 4. Dashed lines correspond to future experimental prospects. A benchmark scenario where $a_{i j}^{d}=v_{i j}^{d} \simeq c_{d} V_{t i} V_{t j}^{*}$, with $c_{d} \approx 10^{-2}$, has been considered, while the ALP width is fixed to an illustrative value $\Gamma_{a}=10^{-6} \mathrm{GeV}$. Larger (smaller) values of $\Gamma_{a}$ would decrease (increase) the excluded regions in the on-shell regions for semileptonic decays. Constraints on $\left|s_{31}^{\ell}\right|$ are not depicted, since they turn out to be very similar to $\left|s_{32}^{\ell}\right|$.

affecting the off-shell ones. We stress that our expressions and experimental/theoretical inputs are given in full generality, so that the correct (resonant) bound could be easily assessed to specific flavor models. For the illustrative case we consider, as shown in figure 13, we see that the most stringent constraints indeed come from semileptonic decays such as $K \rightarrow \pi \mu e, B \rightarrow K^{(*)} \mu e$ and $B \rightarrow K^{(*)} \mu \tau$, in the resonant regions. On the other hand, for small masses the most stringent constraints come from the decay $K_{L} \rightarrow \mu e$. Prospects for existing/future experiments are also shown in figure 13 when available, cf. table 2 .

\section{Conclusions}

In this work, we have explored the signatures of axion-like particles (ALPs) in lepton flavor violating (LFV) observables at low energies. By using the most general dimension-5 effective Lagrangian, which accounts for the ALP couplings to SM fermions and gauge bosons, we have derived complete expressions for the most relevant LFV decays of leptons and hadrons. These general formulae can be applied for any ALP mass, as well as for any choice of ALP couplings, thus generalising and complementing previous results available in the literature [47-49].

Purely leptonic observables comprise the decays $\ell_{j} \rightarrow \ell_{i} \gamma, \ell_{j} \rightarrow \ell_{i} \ell_{k} \ell_{k}$ and $\ell_{j} \rightarrow \ell_{i} \gamma \gamma$ as well as $\mu \rightarrow e$ conversion in nuclei. We find that, currently, the most stringent limits concern the $\mu \rightarrow e$ transition, and arise from the decay modes $\mu \rightarrow 3 e$ and $\mu \rightarrow e \gamma \gamma$ for $m_{a}<m_{\mu}$. For $m_{a}>m_{\mu}$, the most stringent constraint arises from $\mu \rightarrow e \gamma$, which is going to be superseded in the future by the experimental searches for $\mu \rightarrow e$ conversion in nuclei 
at COMET and Mu2E experiments [44, 46]. Likewise, for the $\tau \rightarrow \mu$ and $\tau \rightarrow e$ transitions, the three body decays $\tau \rightarrow 3 \mu$ and $\tau \rightarrow e \mu \mu$ set the most stringent bounds in the range $2 m_{\mu} \lesssim m_{a} \lesssim m_{\tau}$, while the radiative decays are the most constraining modes for $m_{a}>m_{\tau}$. We have fully explored the complementarity of the different decay modes by showing, in particular, that tree-level contributions to $\ell_{j} \rightarrow \ell_{i} \ell_{k} \ell_{k}$ are not entirely negligible above the threshold of on-shell ALP production, and by estimating these contributions along with their interference with loop-level contributions, see figure 5. Furthermore, we show that the ALP mass can be inferred from the correlation among the different leptonic processes, as illustrated, for instance, by comparing $\mathcal{B}(\mu \rightarrow e \gamma)$ with $\mathcal{B}(\mu \rightarrow 3 e)$ and $\mathcal{B}(\mu+N \rightarrow e+N)$ in figures 5 and 6 , respectively.

Concerning hadronic processes, we have focused on the leptonic decays $P \rightarrow \ell_{i} \ell_{j}$ and $\tau \rightarrow P \ell_{j}$, and the semileptonic ones $P \rightarrow P^{\prime}(V) \ell_{i} \ell_{j}$, where $P^{(\prime)}$ and $V$ stand for pseudoscalar and vector mesons, respectively. Leptonic decays can be induced via ALP couplings to quarks and/or gluons. We find that $\tau$ decays into light unflavored mesons $\left(\pi^{0}\right.$, $\eta$ and $\eta^{\prime}$ ) are the most sensitive probes of the gluonic coupling, while decays of kaons and $B$-mesons are particularly sensitive to quark-flavor violating ALP interactions. On other hand, semileptonic decays can only probe the vector or axial ALP couplings, depending on the spin of the meson in the final state. For this reason, these processes are very complementary probes of ALP interactions, as we have made explicit for a benchmark scenario in figure 13.

As a by-product of this study, we have also revisited ALP contributions to the anomalous magnetic moment of leptons, and we have reassessed the possibility of simultaneously explaining the observed discrepancies in $(g-2)_{e}$ and $(g-2)_{\mu}$ via ALP contributions. For flavor conserving contributions, we find that very large ALP couplings to electrons and photons are needed. On the other hand, LFV contributions can be especially relevant for $(g-2)_{e}$ given the large $m_{\tau} / m_{e}$ chiral enhancement at the amplitude level, cf. eq. (3.42). This enhancement can be exploited to provide a more natural explanation of these anomalies, by invoking flavor conserving contributions of Barr-Zee type for $(g-2)_{\mu}$ and LFV effects for $(g-2)_{e}$, see figure 8 . The main prediction of this scenario would be large values of $\mathcal{B}(\tau \rightarrow e \gamma)$, within reach of Belle-II.

In summary, ALPs can induce a plethora of low-energy LFV phenomena with specific patterns that we have identified in this paper. The ongoing experimental program at present NA62 [41], LHCb [42] and Belle-II [43] experiments, as well as the future ones Mu2E [44], Mu3E [45] and COMET [46], plan to improve the current experimental sensitivities by orders of magnitude, offering many possibilities to discover ALPs indirectly through their LFV interactions.

\section{Acknowledgments}

We thank D. Bečirević, F. Feruglio, J. Fuentes-Martin, M. König, F. Mescia and M. Passera for useful discussions. This project has received support by the European Union's Horizon 2020 research and innovation programme under the Marie Sklodowska-Curie grant agreement $\mathrm{N}^{\circ} 674896$ (ITN Elusives), by the exchange of researchers project "The flavor of the 
invisible universe" funded by the Italian Ministry of Foreign Affairs and International Cooperation (MAECI), and by the Swiss National Science Foundation (SNF) under contract 200021-159720.

\section{A Useful formulas}

\section{A.1 Loop functions}

In this appendix, we provide the explicit expressions for the loop functions appearing in section 3 :

$$
\begin{aligned}
& g_{\gamma}(x)=2 \ln \frac{\Lambda^{2}}{m_{a}^{2}}-\frac{\log x}{x-1}-(x-1) \log \frac{x}{x-1}-2, \\
& g_{1}(x)=\frac{x-3}{x-1} x^{2} \log x+1-2 x-2 x^{\frac{3}{2}} \sqrt{x-4} \log \left(\frac{\sqrt{x}+\sqrt{x-4}}{2}\right), \\
& g_{2}(x)=1-2 x+2(x-1) x \log \frac{x}{x-1}, \\
& g_{3}(x)=\frac{2 x^{2} \log x}{(x-1)^{3}}+\frac{1-3 x}{(x-1)^{2}}, \\
& g_{4}(x)=1-2 x+2(x-1) x \log \frac{x}{x-1}, \\
& h_{1}(x)=1+2 x-(x-1) x \log x+2 x(x-3) \sqrt{\frac{x}{x-4}} \log \left(\frac{\sqrt{x}+\sqrt{x-4}}{2}\right), \\
& h_{2}(x)=1+\frac{x^{2}}{6} \log x-\frac{x}{3}-\frac{x+2}{3} \sqrt{(x-4) x} \log \left(\frac{\sqrt{x}+\sqrt{x-4}}{2}\right), \\
& h_{3}(x)=2 x^{2} \log \frac{x}{x-1}-1-2 x .
\end{aligned}
$$

\section{A.2 $\quad \ell_{j} \rightarrow \ell_{i} \ell_{k} \ell_{k}$ kinematic functions}

The $\ell_{j} \rightarrow \ell_{i} \ell_{k} \ell_{k}$ phase-space functions that appear in eq. (3.19) and (3.20) are given by

$$
\begin{aligned}
& \varphi_{0}^{i i}(x)=-\frac{11}{4}+4 x- {\left[\frac{x^{2}}{2} \log \frac{2 x-1}{x}-1+5 x-4 x^{2}\right] \log \frac{x-1}{x} } \\
&+\frac{x^{2}}{2}\left[\operatorname{Li}_{2}\left(\frac{x-1}{2 x-1}\right)-\operatorname{Li}_{2}\left(\frac{x}{2 x-1}\right)\right], \\
& \varphi_{0}^{i \neq k}(x)=\left(3 x^{2}-4 x+1\right) \log \frac{x-1}{x}+3 x-\frac{5}{2}, \\
& \varphi_{1}(x)=-3+6 x+6(x-1) x \log \frac{x-1}{x}, \\
& \varphi_{2}(x)=2-2(x-1) \varphi_{1}(x),
\end{aligned}
$$

where we remind the reader that $x=m_{a}^{2} / m_{\ell_{j}}$. In the limit of heavy ALP masses, these expressions can be simplified as $\varphi_{0}^{i i}(x)=1 /\left(16 x^{2}\right)+\mathcal{O}\left(1 / x^{3}\right), \varphi_{0}^{i \neq k}(x)=1 /\left(12 x^{2}\right)+\mathcal{O}\left(1 / x^{3}\right)$ and $\varphi_{1,2}(x)=1 / x+\mathcal{O}\left(1 / x^{2}\right)$. 


\section{A.3 Form factors for $\ell_{j} \rightarrow \ell_{i} \gamma^{*}$}

In this appendix we collect the complete one-loop form factors for $\ell_{j} \rightarrow \ell_{i} \gamma^{*}$ in terms of the Passarino Veltman (PaVe) functions, as defined in the PACKAGE-X documentation [70]. The photon 4-momentum is denoted as $q$ and we use the following shorthand notation for the ALP coupling to leptons,

$$
A_{a b} \equiv\left(m_{b}+m_{a}\right) a_{a b}^{\ell}, \quad V_{a b} \equiv\left(m_{b}-m_{a}\right) v_{a b}^{\ell},
$$

where $m_{i} \equiv m_{\ell_{i}}$. The three-point PaVe appearing in our expressions have three possible arguments, which we denote as

$$
\begin{aligned}
& \theta_{3}=\left\{m_{j}^{2}, m_{i}^{2}, q^{2} ; m_{k}, m_{a}, m_{k}\right\}, \\
& \theta_{2}=\left\{m_{j}^{2}, q^{2}, m_{i}^{2} ; m_{a}, m_{k}, m_{k}\right\}, \\
& \theta_{1}=\left\{m_{j}^{2}, m_{i}^{2}, q^{2} ; 0, m_{j}, m_{a}\right\}, \\
& \theta_{0}=\left\{m_{j}^{2}, m_{i}^{2}, q^{2} ; m_{a}, m_{i}, 0\right\} .
\end{aligned}
$$

The results provided below can be evaluated by using, for instance, Package-X [70].

\section{A.3.1 Linear contributions}

For the contributions that are linear on ALP Yukawas, as depicted in the left panel of figure 1 , we obtain that the anapole form-factors read

$$
\begin{aligned}
& \mathcal{F}_{1}\left(q^{2}\right)=-q^{2} \frac{e^{2} c_{\gamma \gamma} A_{i j}}{8 \pi^{2} \Lambda^{2}}(D-3)\left\{\left(m_{j}-m_{i}\right)\left[\mathcal{C}_{1}\left(\theta_{0}\right)+\mathcal{C}_{12}\left(\theta_{1}\right)+C_{12}\left(\theta_{0}\right)\right]\right. \\
&\left.+m_{j}\left[\mathcal{C}_{11}\left(\theta_{1}\right)+\mathcal{C}_{11}\left(\theta_{0}\right)\right]\right\}, \\
& \mathcal{G}_{1}\left(q^{2}\right)=+q^{2} \frac{e^{2} c_{\gamma \gamma} V_{i j}}{8 \pi^{2} \Lambda^{2}}(D-3)\{\left(m_{j}+m_{i}\right)\left[\mathcal{C}_{1}\left(\theta_{0}\right)+\mathcal{C}_{12}\left(\theta_{1}\right)+C_{12}\left(\theta_{0}\right)\right] \\
&\left.+m_{j}\left[\mathcal{C}_{11}\left(\theta_{1}\right)+\mathcal{C}_{11}\left(\theta_{0}\right)\right]\right\},
\end{aligned}
$$

which vanish at $q^{2}=0$, as expected by gauge invariance. Similarly, for the dipole formfactors we obtain

$$
\begin{aligned}
\mathcal{F}_{2}\left(q^{2}\right)= & -m_{j} \frac{e^{2} c_{\gamma \gamma} A_{i j}}{8 \pi^{2} \Lambda^{2}}(D-3)\left\{\left(m_{j}^{2}-m_{i}^{2}\right)\left[C_{1}\left(\theta_{0}\right)+\mathcal{C}_{12}\left(\theta_{1}\right)+C_{12}\left(\theta_{0}\right)\right]\right. \\
& \left.+m_{j}\left(m_{j}+m_{i}\right)\left[\mathcal{C}_{11}\left(\theta_{1}\right)+\mathcal{C}_{11}\left(\theta_{0}\right)\right]+2(D-2)\left[\mathcal{C}_{00}\left(\theta_{0}\right)+\mathcal{C}_{00}\left(\theta_{1}\right)\right]\right\}, \\
\mathcal{G}_{2}\left(q^{2}\right)= & -m_{j} \frac{e^{2} c_{\gamma \gamma} V_{i j}}{8 \pi^{2} \Lambda^{2}}(D-3)\left\{\left(m_{j}^{2}-m_{i}^{2}\right)\left[C_{1}\left(\theta_{0}\right)+\mathcal{C}_{12}\left(\theta_{1}\right)+C_{12}\left(\theta_{0}\right)\right]\right. \\
& \left.+m_{j}\left(m_{j}-m_{i}\right)\left[\mathcal{C}_{11}\left(\theta_{1}\right)+\mathcal{C}_{11}\left(\theta_{0}\right)\right]+2(D-2)\left[\mathcal{C}_{00}\left(\theta_{0}\right)+\mathcal{C}_{00}\left(\theta_{1}\right)\right]\right\} .
\end{aligned}
$$

These expressions have been obtained within dimensional regularization, in a scheme where the Levi-Civita symbol is a $D$-dimensional object [106]. The scheme choice affects the finite terms for the dipole form-factors since they are UV-sensitive, see discussion in ref. [30]. 


\section{A.3.2 Quadratic contributions}

For the contributions that are quadratic on ALP Yukawas, as depicted in the right panel of figure 1, we obtain that the anapole form-factors are given by

$$
\begin{aligned}
& \mathcal{F}_{1}\left(q^{2}\right)=\frac{1}{16 \pi^{2} \Lambda^{2}}\left[\left(A_{i k} A_{k j}+V_{i k} V_{k j}\right) \mathcal{F}_{1}^{+}\left(q^{2}\right)+\left(A_{i k} A_{k j}-V_{i k} V_{k j}\right) \mathcal{G}_{1}^{-}\left(q^{2}\right)\right], \\
& \mathcal{G}_{1}\left(q^{2}\right)=\frac{1}{16 \pi^{2} \Lambda^{2}}\left[\left(V_{i k} A_{k j}+A_{i k} V_{k j}\right) \mathcal{F}_{1}^{+}\left(q^{2}\right)+\left(V_{i k} A_{k j}-A_{i k} V_{k j}\right) \mathcal{G}_{1}^{-}\left(q^{2}\right)\right],
\end{aligned}
$$

where

$$
\begin{aligned}
\mathcal{F}_{1}^{+}\left(q^{2}\right)= & m_{k}\left(m_{j}+m_{i}\right) \mathcal{C}_{1}\left(\theta_{3}\right)+\frac{m_{k}}{m_{j}-m_{i}}\left[\mathcal{B}_{0}\left(m_{i}^{2} ; m_{a}, m_{k}\right)-\mathcal{B}_{0}\left(m_{j}^{2} ; m_{a}, m_{k}\right)\right], \\
\mathcal{F}_{1}^{-}\left(q^{2}\right)= & 2 \mathcal{C}_{00}\left(\theta_{3}\right)+m_{j}\left(m_{j}+m_{i}\right) \mathcal{C}_{11}\left(\theta_{3}\right)+\frac{A_{0}\left(m_{k}\right)-A_{0}\left(m_{a}\right)}{2 m_{i} m_{j}}-\mathcal{B}_{0}\left(q^{2} ; m_{k}, m_{k}\right) \\
& -\frac{m_{a}^{2}-m_{i}^{2}-m_{k}^{2}}{2 m_{i}\left(m_{i}-m_{j}\right)} \mathcal{B}_{0}\left(m_{i}^{2} ; m_{a}, m_{k}\right)-\frac{m_{a}^{2}-m_{j}^{2}-m_{k}^{2}}{2 m_{j}\left(m_{j}-m_{i}\right)} \mathcal{B}_{0}\left(m_{j}^{2} ; m_{a}, m_{k}\right) \\
& -\left(m_{a}^{2}-m_{j}^{2}-m_{k}^{2}\right) \mathcal{C}_{0}\left(\theta_{2}\right)+\left(m_{j}^{2}-m_{i}^{2}\right)\left[\mathcal{C}_{12}\left(\theta_{3}\right)+\mathcal{C}_{2}\left(\theta_{3}\right)\right]+2 m_{j}^{2} \mathcal{C}_{1}\left(\theta_{3}\right),
\end{aligned}
$$

while $\mathcal{G}_{1}^{ \pm}\left(q^{2}\right)$ is given by $\pm \mathcal{F}_{1}^{ \pm}\left(q^{2}\right)$ with the replacement $m_{i} \rightarrow-m_{i}$. Lastly, the dipole form-factors read

$$
\begin{aligned}
\mathcal{F}_{2}\left(q^{2}\right)=\frac{m_{j}}{16 \pi^{2} \Lambda^{2}}\{ & \left(A_{i k} A_{k j}-V_{i k} V_{k j}\right)\left[m_{j} C_{11}\left(\theta_{2}\right)+m_{i} C_{22}\left(\theta_{2}\right)+\left(m_{j}+m_{i}\right) C_{12}\left(\theta_{2}\right)\right] \\
- & {\left[\left(m_{k}-m_{i}\right) A_{i k} A_{k j}+\left(m_{k}+m_{i}\right) V_{i k} V_{k j}\right] C_{2}\left(\theta_{2}\right) } \\
- & {\left.\left[\left(m_{k}-m_{j}\right) A_{i k} A_{k j}+\left(m_{k}+m_{j}\right) V_{i k} V_{k j}\right] C_{1}\left(\theta_{2}\right)\right\}, } \\
\mathcal{G}_{2}\left(q^{2}\right)=\frac{m_{j}}{16 \pi^{2} \Lambda^{2}}\{ & \left(V_{i k} A_{k j}-A_{i k} V_{k j}\right)\left[m_{j} C_{11}\left(\theta_{2}\right)-m_{i} C_{22}\left(\theta_{2}\right)+\left(m_{j}-m_{i}\right) C_{12}\left(\theta_{2}\right)\right] \\
- & {\left[\left(m_{k}+m_{i}\right) V_{i k} A_{k j}+\left(m_{k}-m_{i}\right) A_{i k} V_{k j}\right] C_{2}\left(\theta_{2}\right) } \\
- & {\left.\left[\left(m_{k}-m_{j}\right) V_{i k} A_{k j}+\left(m_{k}+m_{j}\right) A_{i k} V_{k j}\right] C_{1}\left(\theta_{2}\right)\right\} . }
\end{aligned}
$$

\section{A.4 Taylor-expanded anapole form factors}

For $m_{a}>m_{\ell_{j}}$, it is a good approximation to Taylor expand the form factors $\mathcal{F}_{1}$ and $\mathcal{G}_{1}$ around $q^{2}=0$, cf. eq. (3.16). In this appendix, we provide the explicit expression for the $q^{2}$-derivative of $\mathcal{F}_{1}\left(q^{2}\right)$ and $\mathcal{G}_{1}\left(q^{2}\right)$ evaluated at $q^{2}=0$. 


\section{A.4.1 Linear contributions}

For the contributions illustrated in the right panel of figure 1, we obtain

$$
\begin{aligned}
\dot{\mathcal{F}}_{1}(0)=\frac{a_{j i}^{\ell} c_{\gamma \gamma} \alpha_{\mathrm{em}}}{6 \pi \Lambda^{2}} & {\left[6 x_{j}^{2} \operatorname{Li}_{2}\left(\frac{x_{j}-1}{x_{j}}\right)-\pi^{2} x_{j}^{2}+3\left(x_{j}+1\right)+3\left(x_{j}-1\right) x_{j} \log \frac{x_{j}}{x_{j}-1}\right.} \\
& \left.+3 x \frac{2 x_{j}-1}{x_{j}-1} \log x_{j}\right],
\end{aligned}
$$

while the axial form-factor reads $\dot{\mathcal{G}}_{1}(0)=-\dot{\mathcal{F}}_{1}(0) \frac{v_{j i}^{\ell}}{a_{j i}^{\ell}}$.

\section{A.4.2 Quadratic contributions}

Similarly to the discussion in section 3.1 for the dipole operators, the expression for the chirality-conserving form-factors will depend on the mass of the particle running in the loop, as described in the following:

- For $j=k>i$, we obtain that

$$
\begin{aligned}
\dot{\mathcal{F}}_{1}(0)= & -\frac{a_{i j}^{\ell} a_{j j}^{\ell}}{16 \pi^{2} \Lambda^{2}}\left\{2 x_{j} \frac{2 x_{j}^{2}-5 x_{j}+2}{x_{j}-1} \log x_{j}+4 m_{j}^{2}\left(x_{j}-1\right)^{2} \mathcal{C}_{0}\left(0,0, m_{j}^{2} ; m_{a}, m_{j}, m_{j}\right)\right. \\
& \left.+7-12 x_{j}-4\left(2 x_{j}-1\right) \sqrt{\left(x_{j}-4\right) x_{j}} \log \left(\frac{\sqrt{x_{j}}+\sqrt{x_{j}-4}}{2}\right)\right\}, \\
\dot{\mathcal{G}}_{1}(0)= & -\dot{\mathcal{F}}_{1}(0) \frac{v_{j i}^{\ell}}{a_{j i}^{\ell}}
\end{aligned}
$$

- Similarly, for $j>k=i$, we obtain that

$$
\begin{aligned}
\dot{\mathcal{F}}_{1}(0)= & \frac{a_{i i}^{\ell} a_{i j}^{\ell}}{8 \pi^{2} \Lambda^{2}} \frac{m_{i}}{m_{j}}\left\{\left(4 x_{j}^{2}-5 x_{j}+1\right) \log \frac{x_{j}-1}{x_{j}}+6 x_{j}+\left(1-2 x_{j}\right) \log x_{j}-\frac{5}{2}\right. \\
& \left.+2 m_{j}^{2} x_{j}\left(1-x_{j}\right) \mathcal{C}_{0}\left(0, m_{i}^{2}, m_{j}^{2} ; m_{i}, m_{i}, m_{a}\right)+\left(2 x_{j}-1\right) \log \frac{m_{i}^{2}}{m_{j}^{2}}\right\}
\end{aligned}
$$

- For $\mu \rightarrow e \gamma$, there is an additional contribution from $\tau$-loops (i.e. $k>j>i$ in our notation), which is given by

$$
\begin{aligned}
& \dot{\mathcal{F}}_{1}(0)=-\frac{a_{\tau e} a_{\tau \mu}+v_{\tau e} v_{\tau \mu}}{32 \pi^{2} \Lambda^{2}} f_{1}\left(x_{k}\right), \\
& \dot{\mathcal{G}}_{1}(0)=+\frac{v_{\tau e} a_{\tau \mu}+a_{\tau e} v_{\tau \mu}}{32 \pi^{2} \Lambda^{2}} f_{1}\left(x_{k}\right),
\end{aligned}
$$

where $h_{4}(x)$ is given by

$$
f_{1}(x)=\frac{-16 x^{3}+45 x^{2}+6(2 x-3) x^{2} \log x-36 x+7}{18(x-1)^{4}} .
$$


- Lastly, $\tau \rightarrow \mu \gamma$ might receive a contribution from electrons running in the loop. In this case,

$$
\begin{aligned}
& \dot{\mathcal{F}}_{1}(0)=\frac{a_{i k}^{\ell} a_{k j}^{\ell}+v_{i k}^{\ell} v_{k j}^{\ell}}{32 \pi^{2} \Lambda^{2}} \frac{m_{i}}{m_{j}} f_{2}\left(x_{j}\right), \\
& \dot{\mathcal{G}}_{1}(0)=\frac{v_{i k}^{\ell} a_{k j}^{\ell}+v_{k j}^{\ell} a_{i k}^{\ell}}{32 \pi^{2} \Lambda^{2}} \frac{m_{i}}{m_{j}} f_{2}\left(x_{j}\right),
\end{aligned}
$$

with

$$
\begin{aligned}
f_{2}(x)= & (2-4 x) \log (x-1)+x(6-8 x) \log \frac{x}{x-1}+(4 x-2) \log \frac{m_{k}^{2}}{m_{j}^{2}} \\
& +12 x-5-4 x(x-1) m_{j}^{2} \mathcal{C}_{0}\left(0,0, m_{j}^{2} ; m_{k}, m_{k}, m_{a}\right) .
\end{aligned}
$$

For compactness, we left explicit the dependence on the PaVe function $\mathcal{C}_{0}$ in the above expressions. This function can be evaluated by using the integral form

$$
\mathcal{C}_{0}\left(r_{10}^{2}, r_{12}^{2}, r_{20}^{2} ; m_{0}^{2}, m_{1}^{2}, m_{2}^{2}\right)=-\int_{0}^{1} \mathrm{~d} x \int_{0}^{1-y} \mathrm{~d} x \frac{1}{\Delta(x, y)},
$$

with

$\Delta(x, y)=(1-x-y) m_{0}^{2}+x m_{1}^{2}+y m_{2}^{2}-x(1-x) r_{10}^{2}-y(1-y) r_{20}^{2}+x y\left(r_{10}^{2}+r_{20}^{2}-r_{21}^{2}\right)$, or, alternatively, by using PACKAGE-X [70].

Open Access. This article is distributed under the terms of the Creative Commons Attribution License (CC-BY 4.0), which permits any use, distribution and reproduction in any medium, provided the original author(s) and source are credited.

\section{References}

[1] J. Jaeckel and A. Ringwald, The Low-Energy Frontier of Particle Physics, Ann. Rev. Nucl. Part. Sci. 60 (2010) 405 [arXiv: 1002. 0329] [INSPIRE].

[2] A. Arvanitaki, S. Dimopoulos, S. Dubovsky, N. Kaloper and J. March-Russell, String Axiverse, Phys. Rev. D 81 (2010) 123530 [arXiv:0905.4720] [InSPIRE].

[3] I.G. Irastorza and J. Redondo, New experimental approaches in the search for axion-like particles, Prog. Part. Nucl. Phys. 102 (2018) 89 [arXiv:1801.08127] [inSPIRE].

[4] R.D. Peccei and H.R. Quinn, CP Conservation in the Presence of Instantons, Phys. Rev. Lett. 38 (1977) 1440 [INSPIRE].

[5] S. Weinberg, A New Light Boson?, Phys. Rev. Lett. 40 (1978) 223 [InSPIRE].

[6] F. Wilczek, Problem of Strong $P$ and T Invariance in the Presence of Instantons, Phys. Rev. Lett. 40 (1978) 279 [INSPIRE].

[7] M. Gorghetto, E. Hardy and G. Villadoro, Axions from Strings: the Attractive Solution, JHEP 07 (2018) 151 [arXiv: 1806.04677] [INSPIRE]. 
[8] J. Preskill, M.B. Wise and F. Wilczek, Cosmology of the Invisible Axion, Phys. Lett. B 120 (1983) 127 [INSPIRE].

[9] L.F. Abbott and P. Sikivie, A Cosmological Bound on the Invisible Axion, Phys. Lett. B 120 (1983) 133 [INSPIRE].

[10] M. Dine and W. Fischler, The Not So Harmless Axion, Phys. Lett. B 120 (1983) 137 [INSPIRE].

[11] R.L. Davis, Cosmic Axions from Cosmic Strings, Phys. Lett. B 180 (1986) 225 [InSPIRE].

[12] G. Dvali and A. Vilenkin, Cosmic attractors and gauge hierarchy, Phys. Rev. D 70 (2004) 063501 [hep-th/0304043] [INSPIRE].

[13] P.W. Graham, D.E. Kaplan and S. Rajendran, Cosmological Relaxation of the Electroweak Scale, Phys. Rev. Lett. 115 (2015) 221801 [arXiv:1504.07551] [INSPIRE].

[14] F. Wilczek, Axions and Family Symmetry Breaking, Phys. Rev. Lett. 49 (1982) 1549 [INSPIRE].

[15] A. Davidson and K.C. Wali, Minimal Flavor Unification Via Multigenerational Peccei-Quinn Symmetry, Phys. Rev. Lett. 48 (1982) 11 [INSPIRE].

[16] Y. Ema, K. Hamaguchi, T. Moroi and K. Nakayama, Flaxion: a minimal extension to solve puzzles in the standard model, JHEP 01 (2017) 096 [arXiv: 1612.05492] [INSPIRE].

[17] L. Calibbi, F. Goertz, D. Redigolo, R. Ziegler and J. Zupan, Minimal axion model from flavor, Phys. Rev. D 95 (2017) 095009 [arXiv: 1612.08040] [INSPIRE].

[18] H. Georgi, D.B. Kaplan and L. Randall, Manifesting the Invisible Axion at Low-energies, Phys. Lett. B 169 (1986) 73 [INSPIRE].

[19] L. Di Luzio, F. Mescia and E. Nardi, Redefining the Axion Window, Phys. Rev. Lett. 118 (2017) 031801 [arXiv:1610.07593] [INSPIRE].

[20] J. Jaeckel and M. Spannowsky, Probing MeV to $90 \mathrm{GeV}$ axion-like particles with LEP and LHC, Phys. Lett. B 753 (2016) 482 [arXiv: 1509.00476] [INSPIRE].

[21] S. Knapen, T. Lin, H.K. Lou and T. Melia, Searching for Axionlike Particles with Ultraperipheral Heavy-Ion Collisions, Phys. Rev. Lett. 118 (2017) 171801 [arXiv: 1607.06083] [INSPIRE].

[22] T. Flacke, C. Frugiuele, E. Fuchs, R.S. Gupta and G. Perez, Phenomenology of relaxion-Higgs mixing, JHEP 06 (2017) 050 [arXiv:1610.02025] [INSPIRE].

[23] I. Brivio et al., ALPs Effective Field Theory and Collider Signatures, Eur. Phys. J. C 77 (2017) 572 [arXiv: 1701.05379] [INSPIRE].

[24] J.L. Feng, I. Galon, F. Kling and S. Trojanowski, Axionlike particles at FASER: The LHC as a photon beam dump, Phys. Rev. D 98 (2018) 055021 [arXiv: 1806.02348] [INSPIRE].

[25] M. Bauer, M. Heiles, M. Neubert and A. Thamm, Axion-Like Particles at Future Colliders, Eur. Phys. J. C 79 (2019) 74 [arXiv:1808.10323] [inSPIRE].

[26] X. Cid Vidal, A. Mariotti, D. Redigolo, F. Sala and K. Tobioka, New Axion Searches at Flavor Factories, JHEP 01 (2019) 113 [arXiv:1810.09452] [INSPIRE].

[27] L. Harland-Lang, J. Jaeckel and M. Spannowsky, A fresh look at ALP searches in fixed target experiments, Phys. Lett. B 793 (2019) 281 [arXiv:1902.04878] [INSPIRE]. 
[28] D. Aloni, C. Fanelli, Y. Soreq and M. Williams, Photoproduction of Axionlike Particles, Phys. Rev. Lett. 123 (2019) 071801 [arXiv: 1903.03586] [INSPIRE].

[29] M.B. Gavela, J.M. No, V. Sanz and J.F. de Trocóniz, Non-Resonant Searches for Axion-Like Particles at the LHC, arXiv:1905.12953 [INSPIRE].

[30] M. Bauer, M. Neubert and A. Thamm, Collider Probes of Axion-Like Particles, JHEP 12 (2017) 044 [arXiv: 1708.00443] [INSPIRE].

[31] M.J. Dolan, F. Kahlhoefer, C. McCabe and K. Schmidt-Hoberg, A taste of dark matter: Flavour constraints on pseudoscalar mediators, JHEP 03 (2015) 171 [Erratum JHEP 07 (2015) 103] [arXiv:1412.5174] [INSPIRE].

[32] M.J. Dolan, T. Ferber, C. Hearty, F. Kahlhoefer and K. Schmidt-Hoberg, Revised constraints and Belle II sensitivity for visible and invisible axion-like particles, JHEP 12 (2017) 094 [arXiv: 1709.00009] [INSPIRE].

[33] L. Merlo, F. Pobbe, S. Rigolin and O. Sumensari, Revisiting the production of ALPs at B-factories, JHEP 06 (2019) 091 [arXiv: 1905.03259] [INSPIRE].

[34] B. Batell, M. Pospelov and A. Ritz, Multi-lepton Signatures of a Hidden Sector in Rare B Decays, Phys. Rev. D 83 (2011) 054005 [arXiv:0911.4938] [INSPIRE].

[35] M. Freytsis, Z. Ligeti and J. Thaler, Constraining the Axion Portal with $B \rightarrow K l^{+} l^{-}$, Phys. Rev. D 81 (2010) 034001 [arXiv:0911.5355] [INSPIRE].

[36] E. Izaguirre, T. Lin and B. Shuve, Searching for Axionlike Particles in Flavor-Changing Neutral Current Processes, Phys. Rev. Lett. 118 (2017) 111802 [arXiv:1611.09355] [INSPIRE].

[37] W. Altmannshofer, S. Gori and D.J. Robinson, Constraining axion-like particles from rare pion decays, arXiv:1909.00005 [INSPIRE].

[38] J. Albrecht, E. Stamou, R. Ziegler and R. Zwicky, Probing flavoured Axions in the Tail of $B_{q} \rightarrow \mu^{+} \mu^{-}$, arXiv:1911.05018 [INSPIRE].

[39] M.B. Gavela, R. Houtz, P. Quilez, R. Del Rey and O. Sumensari, Flavor constraints on electroweak ALP couplings, Eur. Phys. J. C 79 (2019) 369 [arXiv:1901.02031] [INSPIRE].

[40] B. Döbrich, F. Ertas, F. Kahlhoefer and T. Spadaro, Model-independent bounds on light pseudoscalars from rare B-meson decays, Phys. Lett. B 790 (2019) 537 [arXiv:1810.11336] [INSPIRE].

[41] NA62 collaboration, Prospects for exotics and LFV at NA62, J. Phys. Conf. Ser. 800 (2017) 012039 [INSPIRE].

[42] LHCb collaboration, Physics case for an LHCb Upgrade II - Opportunities in flavour physics and beyond, in the HL-LHC era, arXiv: 1808.08865 [INSPIRE].

[43] Belle-II collaboration, The Belle II Physics Book, arXiv:1808.10567 [INSPIRE].

[44] Mu2E collaboration, Mu2e Technical Design Report, arXiv:1501.05241 [INSPIRE].

[45] A. Blondel et al., Research Proposal for an Experiment to Search for the Decay $\mu \rightarrow$ eee, arXiv:1301.6113 [INSPIRE].

[46] COMET collaboration, COMET Phase-I Technical Design Report, arXiv:1812.09018 [INSPIRE]. 
[47] J. Heeck and W. Rodejohann, Lepton Flavor Violation with Displaced Vertices, Phys. Lett. B 776 (2018) 385 [arXiv:1710.02062] [INSPIRE].

[48] F. Björkeroth, E.J. Chun and S.F. King, Flavourful Axion Phenomenology, JHEP 08 (2018) 117 [arXiv: 1806.00660] [INSPIRE].

[49] M. Bauer, M. Neubert, S. Renner, M. Schnubel and A. Thamm, Axion-like particles, lepton-flavor violation and a new explanation of $a_{\mu}$ and $a_{e}$, arXiv:1908.00008 [INSPIRE].

[50] W.J. Marciano, A. Masiero, P. Paradisi and M. Passera, Contributions of axionlike particles to lepton dipole moments, Phys. Rev. D 94 (2016) 115033 [arXiv:1607.01022] [INSPIRE].

[51] Muon $g-2$ collaboration, Final Report of the Muon E821 Anomalous Magnetic Moment Measurement at BNL, Phys. Rev. D 73 (2006) 072003 [hep-ex/0602035] [INSPIRE].

[52] A. Keshavarzi, D. Nomura and T. Teubner, Muon $g-2$ and $\alpha\left(M_{Z}^{2}\right)$ : a new data-based analysis, Phys. Rev. D 97 (2018) 114025 [arXiv: 1802.02995] [INSPIRE].

[53] M. Davier, A. Hoecker, B. Malaescu and Z. Zhang, Reevaluation of the hadronic vacuum polarisation contributions to the Standard Model predictions of the muon $g-2$ and $\alpha\left(m_{Z}^{2}\right)$ using newest hadronic cross-section data, Eur. Phys. J. C 77 (2017) 827 [arXiv: 1706. 09436] [INSPIRE].

[54] Muon $g-2$ collaboration, Muon $(g-2)$ Technical Design Report, arXiv:1501.06858 [INSPIRE].

[55] G.F. Giudice, P. Paradisi and M. Passera, Testing new physics with the electron g-2, JHEP 11 (2012) 113 [arXiv:1208.6583] [INSPIRE].

[56] H. Davoudiasl and W.J. Marciano, Tale of two anomalies, Phys. Rev. D 98 (2018) 075011 [arXiv: 1806.10252] [INSPIRE].

[57] F. Feruglio, P. Paradisi and O. Sumensari, Implications of scalar and tensor explanations of $R_{D^{(*)}}$, JHEP 11 (2018) 191 [arXiv:1806.10155] [INSPIRE].

[58] A. Crivellin, M. Hoferichter and P. Schmidt-Wellenburg, Combined explanations of $(g-2)_{\mu, e}$ and implications for a large muon EDM, Phys. Rev. D 98 (2018) 113002 [arXiv: 1807.11484] [INSPIRE].

[59] J. Liu, C.E.M. Wagner and X.-P. Wang, A light complex scalar for the electron and muon anomalous magnetic moments, JHEP 03 (2019) 008 [arXiv:1810.11028] [INSPIRE].

[60] S. Gardner and X. Yan, LIght scalars with lepton number to solve the $(g-2)_{e}$ anomaly, arXiv: 1907.12571 [INSPIRE].

[61] M. Badziak and K. Sakurai, Explanation of electron and muon $g-2$ anomalies in the MSSM, JHEP 10 (2019) 024 [arXiv:1908.03607] [INSPIRE].

[62] G. Hiller, C. Hormigos-Feliu, D.F. Litim and T. Steudtner, Anomalous magnetic moments from asymptotic safety, arXiv:1910.14062 [INSPIRE].

[63] R.H. Parker, C. Yu, W. Zhong, B. Estey and H. Müller, Measurement of the fine-structure constant as a test of the Standard Model, Science 360 (2018) 191 [arXiv:1812.04130] [INSPIRE].

[64] D. Hanneke, S. Fogwell and G. Gabrielse, New Measurement of the Electron Magnetic Moment and the Fine Structure Constant, Phys. Rev. Lett. 100 (2008) 120801 [arXiv:0801.1134] [INSPIRE]. 
[65] D. Hanneke, S.F. Hoogerheide and G. Gabrielse, Cavity Control of a Single-Electron Quantum Cyclotron: Measuring the Electron Magnetic Moment, Phys. Rev. A 83 (2011) 052122 [arXiv: 1009.4831] [INSPIRE].

[66] B.W. Lee, C. Quigg and H.B. Thacker, The Strength of Weak Interactions at Very High-Energies and the Higgs Boson Mass, Phys. Rev. Lett. 38 (1977) 883 [INSPIRE].

[67] B.W. Lee, C. Quigg and H.B. Thacker, Weak Interactions at Very High-Energies: The Role of the Higgs Boson Mass, Phys. Rev. D 16 (1977) 1519 [inSPIRE].

[68] S.M. Barr and A. Zee, Electric Dipole Moment of the Electron and of the Neutron, Phys. Rev. Lett. 65 (1990) 21 [Erratum ibid. 65 (1990) 2920] [INSPIRE].

[69] V. Shtabovenko, R. Mertig and F. Orellana, New Developments in FeynCalc 9.0, Comput. Phys. Commun. 207 (2016) 432 [arXiv:1601.01167] [InSPIRE].

[70] H.H. Patel, Package-X 2.0: A Mathematica package for the analytic calculation of one-loop integrals, Comput. Phys. Commun. 218 (2017) 66 [arXiv:1612.00009] [INSPIRE].

[71] Y. Kuno and Y. Okada, Muon decay and physics beyond the standard model, Rev. Mod. Phys. 73 (2001) 151 [hep-ph/9909265] [INSPIRE].

[72] MEG collaboration, Search for the lepton flavour violating decay $\mu^{+} \rightarrow \mathrm{e}^{+} \gamma$ with the full dataset of the MEG experiment, Eur. Phys. J. C 76 (2016) 434 [arXiv:1605.05081] [INSPIRE].

[73] LHCb collaboration, Search for hidden-sector bosons in $B^{0} \rightarrow K^{* 0} \mu^{+} \mu^{-}$decays, Phys. Rev. Lett. 115 (2015) 161802 [arXiv: 1508.04094] [INSPIRE].

[74] LHCb collaboration, Search for long-lived scalar particles in $B^{+} \rightarrow K^{+} \chi\left(\mu^{+} \mu^{-}\right)$decays, Phys. Rev. D 95 (2017) 071101 [arXiv: 1612.07818] [INSPIRE].

[75] L. Calibbi and G. Signorelli, Charged Lepton Flavour Violation: An Experimental and Theoretical Introduction, Riv. Nuovo Cim. 41 (2018) 71 [arXiv:1709.00294] [INSPIRE].

[76] S. Mihara, J.P. Miller, P. Paradisi and G. Piredda, Charged Lepton Flavor-Violation Experiments, Ann. Rev. Nucl. Part. Sci. 63 (2013) 531 [INSPIRE].

[77] V. Cirigliano, S. Davidson and Y. Kuno, Spin-dependent $\mu \rightarrow e$ conversion, Phys. Lett. B 771 (2017) 242 [arXiv:1703.02057] [INSPIRE].

[78] S. Davidson, Y. Kuno and A. Saporta, "Spin-dependent" $\mu \rightarrow e$ conversion on light nuclei, Eur. Phys. J. C 78 (2018) 109 [arXiv:1710.06787] [INSPIRE].

[79] R. Kitano, M. Koike and Y. Okada, Detailed calculation of lepton flavor violating muon electron conversion rate for various nuclei, Phys. Rev. D 66 (2002) 096002 [Erratum ibid. D 76 (2007) 059902] [hep-ph/0203110] [INSPIRE].

[80] V. Cirigliano, R. Kitano, Y. Okada and P. Tuzon, On the model discriminating power of $\mu \rightarrow e$ conversion in nuclei, Phys. Rev. D 80 (2009) 013002 [arXiv: 0904.0957] [InSPIRE].

[81] SINDRUM II collaboration, A Search for muon to electron conversion in muonic gold, Eur. Phys. J. C 47 (2006) 337 [INSPIRE].

[82] Particle Data Group, Review of Particle Physics, Phys. Rev. D 98 (2018) 030001 [INSPIRE].

[83] TWIST collaboration, Search for two body muon decay signals, Phys. Rev. D 91 (2015) 052020 [arXiv: 1409.0638 ] [INSPIRE]. 
[84] SINDRUM II collaboration, Test of lepton flavor conservation in $\mu \rightarrow e$ conversion on titanium, Phys. Lett. B 317 (1993) 631 [INSPIRE].

[85] ARGUS collaboration, A Search for lepton flavor violating decays $\tau \rightarrow e \alpha, \tau \rightarrow \mu \alpha, Z$. Phys. C 68 (1995) 25 [INSPIRE].

[86] A.M. Baldini et al., MEG Upgrade Proposal, arXiv:1301.7225 [INSPIRE].

[87] J.P. Leveille, The Second Order Weak Correction to (G-2) of the Muon in Arbitrary Gauge Models, Nucl. Phys. B 137 (1978) 63 [INSPIRE].

[88] S. Eidelman and M. Passera, Theory of the tau lepton anomalous magnetic moment, Mod. Phys. Lett. A 22 (2007) 159 [hep-ph/0701260] [INSPIRE].

[89] S. Eidelman, D. Epifanov, M. Fael, L. Mercolli and M. Passera, $\tau$ dipole moments via radiative leptonic $\tau$ decays, JHEP 03 (2016) 140 [arXiv:1601.07987] [INSPIRE].

[90] M. Borsato, V.V. Gligorov, D. Guadagnoli, D. Martinez Santos and O. Sumensari, Effective-field-theory arguments for pursuing lepton-flavor-violating $K$ decays at LHCb, Phys. Rev. D 99 (2019) 055017 [arXiv: 1808.02006] [INSPIRE].

[91] A. Cerri et al., Report from Working Group 4, CERN Yellow Rep. Monogr. 7 (2019) 867 [arXiv: 1812.07638] [INSPIRE].

[92] S. Aoki et al., Review of lattice results concerning low-energy particle physics, Eur. Phys. J. C 77 (2017) 112 [arXiv:1607.00299] [INSPIRE].

[93] D. Bečirević, G. Duplančić, B. Klajn, B. Melić and F. Sanfilippo, Lattice $Q C D$ and $Q C D$ sum rule determination of the decay constants of $\eta_{c}, J / \psi$ and $h_{c}$ states, Nucl. Phys. B 883 (2014) 306 [arXiv:1312.2858] [INSPIRE].

[94] C. McNeile, C.T.H. Davies, E. Follana, K. Hornbostel and G.P. Lepage, Heavy meson masses and decay constants from relativistic heavy quarks in full lattice QCD, Phys. Rev. D 86 (2012) 074503 [arXiv:1207.0994] [INSPIRE].

[95] D.J. Gross, S.B. Treiman and F. Wilczek, Light Quark Masses and Isospin Violation, Phys. Rev. D 19 (1979) 2188 [INSPIRE].

[96] A. Brignole and A. Rossi, Anatomy and phenomenology of mu-tau lepton flavor violation in the MSSM, Nucl. Phys. B 701 (2004) 3 [hep-ph/0404211] [INSPIRE].

[97] T. Feldmann, P. Kroll and B. Stech, Mixing and decay constants of pseudoscalar mesons, Phys. Rev. D 58 (1998) 114006 [hep-ph/9802409] [INSPIRE].

[98] M. Beneke and M. Neubert, Flavor singlet B decay amplitudes in QCD factorization, Nucl. Phys. B 651 (2003) 225 [hep-ph/0210085] [INSPIRE].

[99] D. Bečirević, O. Sumensari and R. Zukanovich Funchal, Lepton flavor violation in exclusive $b \rightarrow s$ decays, Eur. Phys. J. C 76 (2016) 134 [arXiv: 1602.00881] [InSPIRE].

[100] Flavour Lattice Averaging Group, FLAG Review 2019, arXiv:1902.08191 [inSPIRE].

[101] P. Ball and R. Zwicky, New results on $B \rightarrow \pi, K, \eta$ decay formfactors from light-cone sum rules, Phys. Rev. D 71 (2005) 014015 [hep-ph/0406232] [INSPIRE].

[102] A. Bharucha, D.M. Straub and R. Zwicky, $B \rightarrow V \ell^{+} \ell^{-}$in the Standard Model from light-cone sum rules, JHEP 08 (2016) 098 [arXiv: 1503.05534] [INSPIRE].

[103] D. Aloni, Y. Soreq and M. Williams, Coupling QCD-Scale Axionlike Particles to Gluons, Phys. Rev. Lett. 123 (2019) 031803 [arXiv:1811.03474] [INSPIRE]. 
[104] LHCb collaboration, Search for the lepton-flavour violating decays $B^{+} \rightarrow K^{+} \mu^{ \pm} e^{\mp}$, Phys. Rev. Lett. 123 (2019) 241802 [arXiv:1909.01010] [INSPIRE].

[105] LHCb collaboration, Search for the lepton-flavour-violating decays $B_{s}^{0} \rightarrow \tau^{ \pm} \mu^{\mp}$ and $B^{0} \rightarrow \tau^{ \pm} \mu^{\mp}$, Phys. Rev. Lett. 123 (2019) 211801 [arXiv:1905.06614] [INSPIRE].

[106] S.A. Larin, The Renormalization of the axial anomaly in dimensional regularization, Phys. Lett. B 303 (1993) 113 [hep-ph/9302240] [INSPIRE]. 